\title{
A Numerical Study of the Thermal Transient in High-Pressure Diesel Injection
}

\author{
Marco Arienti ${ }^{\dagger}$ \\ Sandia National Laboratories \\ Mark Sussman ${ }^{\ddagger}$ \\ Florida State University
}

$\bar{\dagger}$ Corresponding author: marient@sandia.gov

‡ sussman@math.fsu.edu 


\section{ABSTRACT}

A study of n-dodecane spray atomization, following the prescribed unseating of the injector's needle tip, is presented for a high-pressure, non-cavitating Bosch Diesel injector ("Spray A", in the Engine Combustion Network denomination). In the simulations discussed here, the internal and external multiphase flows are seamlessly calculated across the injection orifice using an interface-capturing approach for the liquid fuel surface together with an embedded boundary formulation for the injector's walls. Another novelty is the capability to model the compressibility of the liquid and the gas phase while maintaining a sharp interface between the two. This setting makes it possible to directly relate time-dependent spray characteristics to the moving internal geometry of the injector and to the exit thermodynamic state of the fuel. Using the Tait's equation of state calibrated for $n$-dodecane, we examine the differences in jet atomization between adiabatic and isothermal wall conditions.

\section{INTRODUCTION}

The atomization process of liquid fuel in direct injection is crucial in determining the fuel vapor-air mixture, but spray model inadequacies are presently a major barrier for the predictive simulation of performance and emissions. One way to achieve progress is to accurately characterize the flow immediately outside of the injector's orifice, so that the correct boundary conditions for turbulent 
combustion can be set in the engine cylinder. Expansion, driven by the large pressure differential, and internal friction, affect the fuel properties at the injector's exit and hence the jet penetration and spray characteristics such as spray angle and size distribution. The rate of injection (ROI) and the rate of momentum (ROM) are key spray model parameters, but flow rate measurements are complicated by non-trivial variations of temperature and density of the liquid fuel inside the injector, coupled to the thermal conditions at the wall. Temperature measurements at the injector's orifice are necessarily intrusive. Meijer et al. (2012) reported the temperature measurements within the sac volume of a dummy Diesel injector without fuel flow performed by different institutions (Caterpillar, Sandia National Laboratories, Eindhoven University of Technology, and IFPEN). Nozzle tip temperatures were found to vary in a range between $360 \mathrm{~K}$ and $390 \mathrm{~K}$ between the different facilities, depending on the methodology used to control the ambient and fuel-injector boundary conditions. It is also not straightforward to extrapolate from nozzle tip temperatures what the fuel temperature is at the orifice because fuel can act as a coolant. And small deviations from the nominal geometry of the nozzle due to manufacturing defects can modify the flow in the sac and introduce further variability in the measurements.

Efforts by the Engine Combustion Network (ECN) to characterize a specific set of injectors at given operational conditions have brought together modelers and experimentalists in the attempt to solve these issues. The results presented here are focused on the role of the aforementioned 
factors in determining the $\mathrm{ROI}$ and $\mathrm{ROM}$ at high injection pressure. With the capabilities brought forward for this study, we are in the position to correlate the change of spray characteristics with fuel flow dynamics inside the injector.

There are currently only a few numerical studies that consider non-isothermal fluids in high-pressure direct injection. In one of the most recent, Salemi et al. (2015) observed a pronounced rise in the exit temperature of the fuel (by tens of degree Kelvin above the reservoir temperature) during the opening and closing transients at an injection pressure of $190 \mathrm{MPa}$. To further study this aspect, we have carried out time-resolved simulations of the gas-liquid interface inside and outside of the injector during the unseating transient of the needle for a single-orifice nozzle.

Our computational approach assumes that the flow is always sub-critical and that evaporation can be neglected, while the remaining physical processes are represented without models for turbulence or atomization. Perhaps the most representative example of such an approach for direct fuel injection is the massive simulation of jet breakup by Shinjo and Umemura (2011), who reached with $6.0 \mathrm{~B}$ cells the grid spacing of $0.35 \mu \mathrm{m}$. That study amounted to a Direct Numerical Simulation of fuel injection, at least within the limits of continuous flow discretization (see Arienti et al., 2011 and references therein for a discussion on sub-micron pinch-off scaling laws). Yet the study by Shinjo and Umemura (2011) was limited to external flow with simple inlet conditions and 
to injection velocities of $100 \mathrm{~m} / \mathrm{s}$ or less. It is difficult to imagine that the resources necessary to carry out similar simulations could become available on a routine basis any time soon. Yet, for a complete study of fuel direct injection it is necessary to also include the effects of nozzle geometry and injection pressures that are more relevant to modern engines. Thus, in this work we consider a large computational domain to include a portion of the injector's tip and allow a substantial jet penetration: the overall length is 170 times the exit orifice diameter. We also consider a much longer simulation time to capture a significant fraction of the needle unseating process; and a fully compressible formulation of the problem, since the exit jet velocity is supersonic with respect to the gas phase. At more than $250 \mathrm{M}$ computational cells, these choices constrain the grid spacing in our simulations to be 10 times larger than in Shinjo and Umemura (2011) for a similar orifice diameter.

Ménard, et al. (2007), Desjardins et al. (2007), and Herrmann (2008), were amongst the first to demonstrate the feasibility of this sort of high-fidelity simulations for direct fuel injection. The role of the injector's geometry in DNS has been considered only more recently. The complete injection cycle of a Diesel injector was covered by Xue et al. (2013) to study the distribution of turbulence and flow parameters at the nozzle exit resulting from the needle movement, with or without wobble. Their calculations were carried out with a Reynolds-Averaged Navier-Stokes (RANS) formulation using a very small domain outside of the injector. With a much larger external domain, 
but in a demonstration without compressibility, Arienti and Sussman $(2012,2014)$ looked at some of the effects on atomization characteristics due to flow turning. Bode et al. (2014) carried out well-resolved Large Eddy Simulations (LES) with sharp-interface capturing: their study focused on the parametric study of the effects of orifice taper ratio on spray characteristics with the incompressible formulation of the multiphase Navier-Stokes equations (for the first three microseconds from injection and in fully unseated, symmetric needle configuration). Battistoni et al. (2015) tested an internal-external flow LES framework focused on the opening and closing transients of the needle, using a barotropic equation of state for the liquid phase and including models for cavitation and condensation as well as standard subgrid models for turbulent viscosity. The main differentiating feature in the present work is the use of an accurate interface-capturing algorithm, so that the liquid surface dynamics and the transient atomization process can be examined more closely; and the inclusion of more realistic thermodynamic properties for the liquid phase.

This paper represents the application of numerical methodologies developed in previous work (in Arienti and Sussman, 2014; Jemison et al., 2014) and the extension of a preliminary study on single-orifice Diesel injector presented in Arienti and Sussman (2015). Although our results will mostly be discussed with respect to a baseline simulation with adiabatic wall boundary conditions $(A B)$, a second case with fixed wall temperature (IB) is shown for comparison. In reality, we 
recognize that a working fuel injector is subject to temperature gradients between the injector body and the nozzle that will not fit either one of these two idealizations.

A novel element of this study is that the setup is prepared for a specific injector (part of the Spray A batch, specimen 210675) from Robert Bosch LLC. To reduce sources of discrepancy between lab measurements and simulations, the model tip of the nozzle was reconstructed based on radiographic tomography data of the hardware. We also exactly implemented the time-dependent needle motion during the injection cycle, including its wobble.

The simulation covers the first $365 \mu$ s from needle activation of this specimen, including the $25 \mu \mathrm{s}$ that follow the appearance of the liquid from the orifice exit and its atomization. While long by DNS standards, this time interval does not include the complete injection transient: Pickett et al. (2013) observed steady-state penetration of the liquid jet in Spray A only after $40 \mu$ s from its appearance. Interestingly, this steady-state injection condition occurs much later than the time necessary for the area of the annular opening (between needle and internal injector's wall) to equal the orifice exit area. According to our calculations, this equivalent annular area corresponds to an average gap of only $1.7 \mu \mathrm{m}$; from that point on, the injector's orifice is where the flow is most restricted. In the following, after highlighting the numerical approach, we focus on the description of the injector model and on the compilation of the properties of $n$-dodecane, as they are necessary for an accurate and consistent description of the liquid phase. The discussion section is divided in 
three parts, with the understanding that they refer to the same two seamless $A B$ and $I B$ simulations: the first part concerns the description of the flow internal to the injector, particularly the transition from zero gap to a partially open configuration; the second part describes the flow characteristics at the exit orifice; and the third discusses features of the liquid jet and forming spray, where subtle differences between the two sprays can be attributed to the dependence of the surface tension coefficient on temperature. Whenever possible, Spray A data from the ECN archive are used to assess the results of the calculations.

\section{NUMERICAL METHOD}

This section synthesizes recent developments on compressible, multiphase flow for high-pressure injection with time-varying geometry and realistic equations of state. The region occupied by material $m=1 \ldots M$ in a computational cell $\Omega$ is such that $\Omega=\bigcup_{m=1}^{M} \Omega_{m}$. The multi-phase compressible Navier-Stokes equations are solved with the mass-, momentum-, and energy-conserving advection algorithm described by Jemison et al. (2014). The solution is advanced in time by a semi-implicit pressure update scheme that asymptotically preserves the standard incompressible pressure projection in the limit of infinite sound speed; see Kwatra et al. (2009) for the original single-phase formulation. This attribute makes interface capturing methods applicable to compressible flows (the user can actually choose which fluid behaves as 
compressible), while using time steps that can be larger compared to typical explicit methods.

For each material we solve the following system of equations:

$$
\begin{aligned}
& \left(F_{m}\right)_{t}+\nabla \cdot\left(F_{m} \mathbf{u}\right)=F_{m} \nabla \cdot \mathbf{u} \\
& \left(\rho_{m} F_{m}\right)_{t}+\nabla \cdot\left(\rho_{m} F_{m} \mathbf{u}\right)=0 \\
& (\rho \mathbf{u})_{t}+\nabla \cdot(\mathbf{u} \otimes \rho \mathbf{u}+p \mathbf{I}-\boldsymbol{\tau})=0 \\
& \left(\rho_{m} E_{m} F_{m}\right)_{t}+\nabla \cdot\left(F_{m}\left(\rho_{m} E_{m} \mathbf{u}+p \mathbf{u}-\mathbf{u} \cdot \boldsymbol{\tau}-k \nabla T\right)\right)=0,
\end{aligned}
$$

where $F_{m}$ is the volume fraction, $E_{m}$ is the total energy, $\rho_{m}$ the phase density, $\tau$ the stress tensor, $p$, $\mathbf{u}$, and $T$ the flow pressure, velocity, and temperature, respectively. The combined density and conductivity are

$$
\begin{aligned}
& \rho=\sum_{m=1}^{M} F_{m} \rho_{m} \\
& k=\sum_{m=1}^{M} F_{m} k_{m} .
\end{aligned}
$$

The stress tensor is

$$
\boldsymbol{\tau}=2 \mu\left(\mathbf{D}-\frac{\mathrm{TR}(\mathbf{D})}{3} \mathbf{I}\right) \quad \mathbf{D}=\frac{\nabla \mathbf{u}+(\nabla \mathbf{u})^{T}}{2},
$$

where $\mu$ is the combined viscosity

$$
\mu=\sum_{m=1}^{M} F_{m} \mu_{m} .
$$

The total energy $E_{m}$ and internal energy $e_{m}$ are related through the equation

$$
E_{m}=\frac{1}{2}|\mathbf{u}|^{2}+e_{m}
$$

The complete solution workflow is detailed in Jemison et al. (2014). After advection and update of 
the conserved variables $(\rho, \rho \mathbf{u}, \rho E)_{m}^{n}$ from time $t^{n}$, the advected velocity $\mathbf{u}^{a}$ is derived from the new state $n+1$ according to the expression

$$
\mathbf{u}^{a}=\frac{\sum_{m=1}^{M}\left|\Omega_{m}^{n+1}\right|(\rho \mathbf{u})_{m}^{n+1}}{\Sigma_{m=1}^{M}\left|\Omega_{m}^{n+1}\right|(\rho)_{m}{ }_{m}+1} .
$$

The "advective pressure," $p^{a}$, and the "advective sound speed," $\left(c^{2}\right)^{a}$ are defined as a function of the advective internal energy and advective density:

$$
\begin{aligned}
& e_{m}^{a}=\frac{(\rho E)_{m}^{n+1}}{(\rho)_{m}^{n+1}}-\frac{1}{2}\left\|\mathbf{u}^{a}\right\|^{2} \\
& p^{a}=P\left(\rho_{m}^{n+1}, e_{m}^{a}\right) \\
& \left(c^{2}\right)^{a}=c^{2}\left(\rho_{m}^{n+1}, e_{m}^{a}\right) .
\end{aligned}
$$

Liquid and gas phases are kept separated in the course of the simulation (here the gas is treated as non-condensable). In computational cells where more than one material's volume fraction is finite, the equation of state of the fluid with the largest volume fraction $F_{\mathrm{m}} \square$ is chosen as "prevailing". Continuity and momentum equilibrium apply at the gas-liquid interface $\Gamma$, of local normal $\mathbf{n}$ and curvature $\kappa$, so that

$$
[\mathbf{n} \cdot(\boldsymbol{\tau}-p \mathbf{I}) \mathbf{n}]=\sigma \kappa \quad \mathbf{x} \in \Gamma
$$

is enforced. However, mass and heat transfer across $\Gamma$ are not allowed at the moment. Heat transfer is enabled at the fluid-solid interface either by direct specification of the heat flux at the solid wall or by assignment of a fixed wall temperature. 
interface. The curvature is evaluated from the level set function by using the method of heights (Sussman and Ohta, 2009). The liquid-gas interface is captured by the ELVIRA method (Pilliod and Puckett, 2004), except for the contact lines at the solid boundary, which are treated by the coupled level-set moment-of-fluid (CLSMOF) method (Jemison et al., 2013). The simple implementation with constant contact angle $\theta_{\mathrm{w}}$ from Arienti and Sussman (2014) is used there. In the CLSMOF algorithm, a piecewise-linear interface reconstruction uses information from the level-set function, the volume-of-fluid function and the phase centroid of the computational cell to produce a slope and an intercept for the local reconstruction of the gas-liquid interface. The extra information on the phase centroid enables the correct partition of multi-material computational cells at a contact line.

The injector wall boundaries are represented with the embedded boundary method described in Arienti and Sussman (2014). The method uses one more signed distance function, the "solid" level set $\psi$ : its magnitude is the minimal distance between the cell center and the surface of the body, and, by convention, its sign is positive outside the body and negative inside it. The evaluation of the boundary normal from the level set function avoids staircase-like artifacts in the solution. When applied to the motion of solid interfaces, the embedded boundary is allowed to sweep the computational Cartesian cells: contact and separation of wall surfaces, such as they occur during the unseating of the injector's needle, are handled in a straightforward and robust 
manner as logical operations on level set function values.

Compared to the formulation in Arienti and Sussman (2014), the fluid-solid coupling strategy used for this paper is made more parallelizable within the distributed memory framework of the Message Passing Interface (MPI) protocol. The starting point is the construction of the solid level set at node centers of an auxiliary $I, J, K$ Cartesian grid. This grid block $\left(l=1 \ldots N_{x} ; J=1 . . N_{y} ; K=1 \ldots N_{z}\right)$ is local to the corresponding MPI process and completely contains its sub-domain, so that from this point on it is possible to discard the geometrical tessellation of the boundary walls while retaining the information that is necessary to calculate $\psi$ (n) auxiliary grid spacing $\Delta x_{s}$ does not depend on the spacing of the grid where the Navier-Stokes equations are discretized, although it should be comparable to it. Slope-limited linear interpolation transfers the signed distance function values from $I, J, K$ to the target point $\mathbf{x}$ on the solution grid. In cases where a solid body rigidly translates without deforming, such as the needle of the injector here, the solid level set function is evaluated only at the beginning of the simulation: for the assigned body displacement at time $t, \mathbf{s}(t)$, the new solid level set function is obtained by interpolating $\psi_{I, J, K}$ at the point $\mathbf{x}-\mathbf{s}(t)$ on the auxiliary grid.

With multiple solid bodies, an equal number of level set functions needs to be evaluated: the final value of $\psi$ is obtained by comparing, with sign, their respective distance functions. In the present case we have two bodies, the needle and the injector's tip (see Figure 2 for part nomenclature), 
but only the needle is moving, therefore

$$
\psi(\mathbf{x})=\min \left(\psi^{\text {needle }}(\mathbf{x}-\mathbf{s}(t)), \psi^{\text {tip }}(\mathbf{x})\right) .
$$

The remaining procedures for populating center and face values in the solid ghost region and for implementing the contact between the gas-liquid interface and the solid wall are as described in Arienti and Sussman (2014).

Another component of our simulation capability is block-structured, adaptive mesh refinement (AMR). The definitions and operators necessary to carry out the AMR tasks are provided by the BoxLib library (CCSE, 2012), which is developed and maintained by the Center for Computational Sciences and Engineering at Lawrence Berkeley National Laboratory to solve partial differential equations on a structured domain. In parallel calculations, BoxLib manages grid blocks in order to optimize the distribution of computational load amongst all the available processes. The coarsest level (level zero) is always the Cartesian box covering the whole simulation domain, including part or all of the solid bodies. At every new re-gridding operation, cell tagging targets the gas-liquid interface and possibly the solid interface, or special features of the flow (shocks, for instance), depending on how the error function is configured. The new level can in turn be tagged for refinement, and the process is repeated until the input grid resolution is achieved (the refinement ratio between two consecutive levels is two.) In the BoxLib implementation, there is no direct parent-child connection between levels of refinement: a fine grid does not necessarily have a 
unique parent grid, so that data can be organized into relatively large aggregate grids to amortize the cost incurred by the irregular nature of adaptive meshes. The minimum number of cells on any block side defines the granularity of the AMR simulation: this value is 32 in this work. At each re-gridding operation, new grids are combined to cover all the tagged cells within the assigned coverage efficiency. Data on the fine level are either copied from a previous time step or, when a new box is added, they are conservatively interpolated from the underlying coarse level. The liquid-gas interface is always embedded in the finest grid level to avoid gross interpolation errors from the calculation of the fluxes of mass, momentum and energy across fine-coarse boundaries. An extensive discussion on this topic can be found in Kadiouglu and Sussman (2008).

\section{INJECTOR MODEL}

\section{INJECTOR PROPERTIES}

Spray A (Figure 1) is a Bosch injector of $90 \mu \mathrm{m}$ nominal diameter at the orifice exit. As with all the other injectors of this batch, the SN 210675 specimen displays slight manufacturing defects that affect the orifice alignment with the injector axis, its cross-section, and the orifice tapering: the reader is referred to the ECN web site for full documentation. In constructing the tessellation of the injector's tip and the needle surface, we reproduced the measured off-center position of the orifice axis with respect to the sac: this displacement is of $53 \mu \mathrm{m}$ at $\theta=+9^{\circ}$ in the axis convention of 
Figure 1. This offset means that the inlet-turning angle is smaller on one side of the orifice compared to the opposite side. Moreover, the outlet area of the orifice exit is $10 \%$ smaller than the nominal value, that is, the effective orifice diameter is only $85 \mu \mathrm{m}$.

The walls of the Diesel injector are the only elements of the solid body that need to be discretized in order to carry out the simulation on the set of Cartesian boxes. The wall tessellation is read at the beginning of the simulation as a list of node coordinates and of node sequences forming triangular faces. To simulate the unseating of the needle, the injector is divided in two parts, the needle tip and the cap, as displayed in Figure 2. The rigid translation of the needle with respect to the injector cap follows an assigned trajectory that includes needle wobble: the three components of the needle trajectory were obtained from high speed X-ray imaging through the metal injector operated in "cold" ambient gas conditions, as tested at the Argonne National Laboratory (Kastengren et al., 2012). In frame (a) of Figure 2 the needle's base is almost completely inside the computational domain (marked by a dashed line), whereas in its fully open position in frame (c) the base of the needle has moved outside. Frame (b) shows an intermediate position and the effect of the deviation from a perfectly axial trajectory: the annular fuel passage is asymmetric at this time.

The trajectory of the needle from Kastengren et al. (2012) is shown in Figure 3 for the $X-Y$ - and $Z$ components (continuous, dashed and dotted line, respectively). Time zero corresponds to the 
instant when the injection command is given: the same reference time is used in our simulations.

The overall recorded time is $5000 \mu \mathrm{s}$, but the needle does not actually move for the first $160 \mu \mathrm{s}$. After that point, substantial lateral displacements can be observed in Figure 3 in addition to the principal axial motion. The peak needle lift is reached after approximately $900 \mu \mathrm{s}$, then the needle reverses direction and closes at approximately $1800 \mu \mathrm{s}$. The corresponding needle velocity is of the order of one meter per second or less. The simulations presented here cover the transient of the needle unseating, corresponding to the first $365 \mu \mathrm{s}$ from needle activation.

\section{PHASE PROPERTIES}

The properties of the two fluids are evaluated at run-time as a function of pressure, phase density and phase internal energy. The thermal conductivities are constant: for air, $k=0.026 \mathrm{~W} / \mathrm{m} \cdot \mathrm{K}$; for $\mathrm{n}$-dodecane, $k=0.157 \mathrm{~W} / \mathrm{m} \cdot \mathrm{K}$; for the metal, $k=17 \mathrm{~W} / \mathrm{m} \cdot \mathrm{K}$. The gas phase is calculated as a perfect gas with the properties of air. The liquid phase is calculated via a set of correlations derived from high-pressure measurements, as explained below.

\section{Equation of state for $n$-dodecane}

The liquid phase pressure, $P(\rho, T)$, is calculated using the Tait formulation with the coefficients proposed by Caudwell et al. (2004) for pressures up to $200 \mathrm{MPa}$ and in the temperature range 298-473 K: 


$$
\rho=\frac{\rho_{0}}{1-A \ln \left[(B+P) /\left(B+P_{0}\right)\right]}
$$

In this and the following relations we maintain the convention of expressing pressure in MPa while the other properties are in SI units. In Equation (16), $A=0.08998$ and $B$ depends only on temperature,

$$
B=\sum_{i=0}^{2} b_{i} T^{i}
$$

with $b_{0}=345.1 \mathrm{MPa}, b_{1}=-1.1458 \mathrm{MPa} \mathrm{K}^{-1}, b_{2}=0.9837 \cdot 10^{-3} \mathrm{MPa} \mathrm{K}^{-2}$. The reference density at $P_{0}$ $=0.1 \mathrm{MPa}$ is

$$
\rho_{0}=921.1984-0.47516 T-3.922 \cdot 10^{-4} T^{2} .
$$

As the correlation for internal energy was not immediately available from the literature, the authors derived a polynomial expression for $e(\square, T)$ in the form

$$
e(P, T)=\sum_{i=0}^{4} g_{i}(P) T^{i},
$$

with

$$
\begin{aligned}
& g_{0}=19.94245 \mathrm{~kJ} / \mathrm{kg}, \\
& g_{1}=2.273845+7.701613 \cdot 10^{-6} P\left(P-P_{0}\right) \quad \mathrm{kJ} / \mathrm{kg} / \mathrm{K}, \\
& g_{2}=-2.279889 \cdot 10^{-3}-3.654273 \cdot 10^{-6} P \quad \mathrm{~kJ} / \mathrm{kg} / \mathrm{K}^{2}, \\
& g_{3}=6.106366 \cdot 10^{-6} \mathrm{~kJ} / \mathrm{kg} / \mathrm{K}^{3},
\end{aligned}
$$




$$
g_{4}=-3.266302 \cdot 10^{-9} \mathrm{~kJ} / \mathrm{kg} / \mathrm{K}^{4} \text {. }
$$

The corrections for pressure in $g_{1}$ and $g_{2}$ are justified by the thermodynamic relation

$$
\left(\frac{\partial e}{\partial P}\right)_{T}=\frac{1}{\rho}\left(\beta_{T} P-\alpha_{P} T\right)
$$

that is used for evaluating

$$
e(P, T) \cong e\left(P_{0}, T\right)+\left(P-P_{0}\right)\left(\frac{\partial e}{\partial P}\right)_{T}
$$

in the fitting procedure. The isobaric expansion coefficient (in $\mathrm{K}^{-1}$ ), $\alpha_{P}$, and the isothermal compressibility (in $\mathrm{MPa}^{-1}$ ), $\beta_{T}$, are tabulated in the range from 0.1 to $140 \mathrm{MPa}$ and from 293.15 to $433.15 \mathrm{~K}$ by Khasanshin et al. (2003). The internal energy from Equation (19) is compared in Figure 4 to NIST data (Lemmon et al., 2015) along isobaric curves at $P=0.1,20$ and $140 \mathrm{MPa}$. Increasing pressures cause the slope of the curves to decrease so that the curves tend to diverge at higher temperature. However, below $400 \mathrm{~K}$ and for pressures up to approximately $100 \mathrm{MPa}$, no substantial effect of pressure on internal energy is noted, that is, the approximation $e(P, T) \cong$ $e(T)$ could be used instead. This simplified relation is useful as initial condition to solve the inverse problem of finding temperature from known internal energy and density, $T(e, \rho)$, at a computational cell; currently the solution is found at run-time by Newton iterations.

\section{Speed of sound}


The speed of sound in the liquid phase is calculated from a separate correlation of temperature and pressure,

$$
\frac{c-c_{0}}{c}=D \ln \left[\frac{E+P}{E+P_{0}}\right]
$$

to avoid taking derivatives of pressure and density correlations. The reference atmospheric speed of sound in equation (27) is

$$
c_{0}=4094-183.21 T^{0.5}+0.07974 T^{1.5}-2.348 \cdot 10^{-6} T^{3} .
$$

The coefficients $D$ and $E$ are a function of temperature, pressure and the carbon number of the fuel (Padilla-Victoria et al., 2013). For pure n-dodecane:

$$
\begin{gathered}
D=0.1652+2.5 \cdot 10^{-3} T-5.85 \cdot 10^{-6} T \cdot P \\
E=-56.91+7.3674 \cdot 10^{-5} T^{2}+0.02260 T+463.5 \cdot \exp (-0.001687 T) .
\end{gathered}
$$

In Equation (29) the expression for $D$ was modified by the authors with respect to the original expression by Padilla-Victoria et al., (2013) to obtain a better match with the values reported by Khasanshin et al. (2003).

\section{Dynamic viscosity}

The dynamic viscosity $\mu(\rho, T)$ is calculated in the range from 298.15 to $473.15 \mathrm{~K}$ and at pressures up to $200 \mathrm{MPa}$ using the correlation by Caudwell et al. (2004):

$$
\mu=\mu^{*} \cdot\left[4.778 \cdot 10^{-9} V^{-2 / 3} M^{1 / 2} T^{1 / 2}\right] \mathrm{Pa} \cdot \mathrm{S}
$$

where $V$ is the molar volume in $\mathrm{m}^{3} \cdot \mathrm{mol}^{-1}$ and $M=0.17034 \mathrm{~kg} \cdot \mathrm{mol}^{-1}$ is the molar mass for 
$\mathrm{n}$-dodecane. The coefficient $\mu^{*}$ is a dimensionless viscosity

$$
\frac{1}{\mu^{*}}=\sum_{i=0}^{3} d_{i}\left(V / V_{0}\right)^{i}
$$

For $n$-dodecane, the coefficients $d_{\mathrm{i}}$ are: $d_{0}=0.321621 ; d_{1}=-0.4803715 ; d_{2}=0.222206 ; d_{3}=$ $-2.9964626 \cdot 10^{-2} . V_{0}$ is a temperature-dependent molar core volume given by

$$
V_{0}=10^{-6} \sum_{i=0}^{3} f_{i} T^{i} \mathrm{~m}^{3} \mathrm{~mol}^{-1} .
$$

The coefficients $f_{\mathrm{i}}$ are $f_{0}=191.54 \mathrm{~m}^{3} \mathrm{~mol}^{-1} ; f_{1}=-0.441338 \mathrm{~m}^{3} \mathrm{~mol}^{-1} \mathrm{~K}^{-1} ; f_{2}=8.98744 \cdot 10^{-4} \mathrm{~m}^{3} \mathrm{~mol}^{-1}$ $\mathrm{K}^{-2} ; f_{3}=-6.7792 \cdot 10^{-7} \mathrm{~m}^{3} \mathrm{~mol}^{-1} \mathrm{~K}^{-3}$.

The smallest value of dynamic viscosity tabulated by Caudwell et al. (2004) is $0.218 \mathrm{mPa} \cdot \mathrm{s}$ at 0.1

$\mathrm{MPa}$ and $T=473.15 \mathrm{~K}$, whereas the largest value is $5.728 \mathrm{mPa} \cdot \mathrm{s}$ at $152.18 \mathrm{MPa}$ and $298.15 \mathrm{~K}$.

This two-orders-of-magnitude variation points to the need to include the dependence of dynamic viscosity on pressure in direct fuel injection simulations. For instance, taking the tabulated values from Caudwell et al. (2004), at $0.1 \mathrm{MPa}$ and $293.15 \mathrm{~K}\left(\rho=746 \mathrm{~kg} / \mathrm{m}^{3}\right.$ and $\left.\mu=1.344 \mathrm{mPa} \cdot \mathrm{s}\right)$, and assuming a fuel exit velocity of $600 \mathrm{~m} / \mathrm{s}$, the Reynolds number based on the orifice diameter would be $R e=30,000$. At the same temperature, but at $150 \mathrm{MPa}$, the dynamic viscosity is larger by more than a factor of four, $\mu=5.728 \mathrm{mPa} \cdot \mathrm{s}$, while the density increases only by $10 \%$, bringing the Reynolds number down to 7,700 . The dependency of $\mu$ on pressure and temperature is presently included in our code. However, we still assume that the spatial variation of $\mu$ is sufficiently small to neglect the corresponding spatial derivative terms in the Navier-Stokes 
equations. In other words, $\nabla \cdot \boldsymbol{\tau} \cong 2 \mu(x) \nabla \cdot\left(\mathbf{D}-\frac{\mathrm{TR}(\mathbf{D})}{3} \mathbf{I}\right)$ in the momentum equation and $\nabla \cdot\left(F_{m} \mathbf{u} \cdot \boldsymbol{\tau}\right) \cong 2 \mu(x) \nabla \cdot\left(F_{m} \mathbf{u} \cdot\left(\mathbf{D}-\frac{\mathrm{TR}(\mathbf{D})}{3} \mathbf{I}\right)\right)$ in the energy equation.

\section{$\underline{\text { Surface tension coefficient }}$}

The surface tension coefficient is modeled in linearized, temperature-dependent form based on the data for n-dodecane published by Queimada et al. (2001) in the interval between $273 \mathrm{~K}$ and $393 \mathrm{~K}:$

$$
\sigma / \mathrm{mN} \cdot \mathrm{m}^{-1}=51.19-0.08832 \mathrm{~T}
$$

Similarly to dynamic viscosity, we have not included the term due to the spatial derivative of $\sigma$ in the Navier-Stokes equation (i.e., no Marangoni force).

The contact angle on the injector's walls is constant, $\theta_{\mathrm{w}}=90^{\circ}$. This choice is motivated by computational convenience. In reality, organic liquids deposited on materials with high specific surface free energy, such as metals, exhibit values of $\cos \theta_{w}$ closer to 1 ; the exact value is a function of the surface treatment, its smoothness, and temperature, amongst other factors (Zisman, 1964). To properly account for these dependencies, the treatment of the contact angle would need to be more advanced than the one proposed in Arienti and Sussman (2014), possibly allowing for the dynamic evaluation of $\theta_{\mathrm{w}}$. Because of the complexity of a dedicated study on the effect of the injector's surface conditions on the contact angle, the most convenient value for $\theta_{w}$ is adopted for these exploratory simulations. 


\section{SIMULATION SETUP}

Calculations were carried out on the Redsky Sandia cluster by using 128 SUN X6275 blades (2.93 GHz dual socket/quad core configuration with $12 \mathrm{~GB}$ RAM per blade) for a total of 512 cores. This is only an average figure, because in this AMR simulation the computational cost increases with the development of the spray outside of the injector. At the beginning of injection, the region of the computational domain occupied by the liquid phase was confined to the sac, but eventually the solution-adaptive mesh refinement algorithm added several more computational grid boxes to continue capturing the liquid surface. Starting with less than 70 millions cells, their count later surpassed 250 millions; in the latest stages of the simulation, more than 256 blades, or 1024 cores, were necessary.

The base grid was a regular Cartesian $64 \times 64 \times 576$ box with the longest side, oriented along the injector's axis, of length $1.53 \mathrm{~cm}$ (170 times the exit orifice diameter). This size is substantially longer than in similar high-fidelity simulations because we intend to follow the jet penetration for a sufficient time period. Three levels of refinement were added to the coarse level in order to obtain a minimum grid spacing of $3.32 \mu \mathrm{m}$, corresponding to 27 computational cells across the orifice diameter. At this grid resolution, the stable time step for the flow of interest (with a supersonic transient in the gas phase) was of the order of three nanoseconds. A fourth level of refinement 
was later added in order to verify grid convergence in the early stage of injection, bringing the cell count to more than $400 \mathrm{M}$ cells. The spacing of the auxiliary grid was set to $\Delta \mathrm{x}_{\mathrm{s}}=9 \mu \mathrm{m}$ for three levels of refinement and to $\Delta \mathrm{x}_{\mathrm{s}}=7 \mu \mathrm{m}$ for four. Finally, the injector representation required a surface mesh (triangular tessellation) of the internal and external walls as well as of the needle: the smallest edge length of this mesh was of $1-2 \mu \mathrm{m}$ at the orifice extremities.

The wall boundary velocities necessary to populate the ghost region inside the solid body were calculated directly by differentiation of the displacement values read from the trajectory file. The fixed injection pressure of $150 \mathrm{MPa}$ was applied directly at the boundary face, as shown Figure 2 . This constant boundary inlet pressure is a convenient simplification: fluctuations of up to $8 \mathrm{MPa}$ over the entire injection cycle have been reported by Pickett et al. (2013), but $150 \mathrm{MPa}$ is a good approximation for the short simulation interval considered here. A fixed exit pressure of $2 \mathrm{MPa}$ was applied to the other five sides of the computational domain box, assuming an open vessel. The velocity field at the six sides of the computational box was set by extrapolation. The initial temperature of the fuel was uniformly set to $343 \mathrm{~K}$ in both the reservoir and the cap. The initial temperature of the gas was set to $303 \mathrm{~K}$. The $303 \mathrm{~K}$ temperature corresponds to the chamber non-evaporating condition used for radiographic measurements of fuel mass at the Argonne National Laboratory (ANL) as described by Pickett et al. (2014). This is not the $900 \mathrm{~K}$ gas temperature of the reference Spray A: in the following, "cold" Spray A data from ANL will be 
preferably used for validation. Finally, in the isothermal wall simulation the wall temperature is fixed at $T_{w}=383 \mathrm{~K}$.

\section{INLET FLOW RATE AND MASS CONSERVATION ASSESSMENT}

The mass-conservation features of the simulations are assessed in more detail in the following.

For the interface-capturing algorithm in compressible flow, a point of reference is the simulation of a molten metal drop impacted by a shock reported by Arienti et al. (2016): in the severe deformation of a drop with 16 cells per initial diameter, the mass variation was only $-0.4 \%$, and it reduced to $+4 \mathrm{e}-04 \%$ with two more levels of refinement. Arguably, these numbers represent a worse case scenario for the current study, because of the larger density ratio and of the shear continuously applied by the crossflow to the molten metal drop.

To assess the fluid-solid coupling algorithm, we start by noting that the inlet flow rate $\dot{m}_{\text {inj }}(t)$ resulting from the pressure boundary condition is not constant in the early phases of needle unseating (in fact, it even takes negative values whenever the gap between the cap and the needle walls temporarily narrows). The amplitude of its variations decreases as the unseating continues and the gap broadens. For three representative time intervals during needle opening, the time history of $\dot{m}_{i n j}(t)$ is plotted as a continuous line in Figure 5 (while the plot is obtained for the $A B$ case, the isothermal case has a very similar behavior). 
The verification of the mass-conserving behavior in this simulation setup requires the time derivative of the total mass of the liquid phase. This value is derived from the differences between consecutive time steps, $d m / d t=\left(m\left(t_{n+1}\right)-m\left(t_{n}\right)\right) /\left(t_{n+1}-t_{n}\right)$ and is shown as scatter plot in Figure 5 : the unfiltered $d m / d t$ displays a substantial amount of noise because both the mass variations and the consecutive time increments are very small. From $\dot{m}_{i n j}$ and $d m / d t$, and taking a control volume that coincides with the computational domain, the liquid phase mass flow rate must equal the time variation of its total mass for mass conservation to be satisfied. This statement holds true until the moment when droplets escape the domain through the outlet boundaries.

In Figure 5, the maximum pointwise difference $\Delta \dot{m}=d m / d t-\dot{m}_{i n j}$ almost reaches $+1 \mathrm{~g} / \mathrm{s}$ at 6 $\mu$ s after the appearance of the jet outside of the injector: as a reference, the measured open-needle value is $2.62 \mathrm{~g} / \mathrm{s}$. We attribute this worst case discrepancy to the small number of computational cells that span the needle gap in this early injection phase. In reality, the instantaneous $\Delta \dot{m}$ can take both positive and negative values (for instance, it is $-0.08 \mathrm{~g} / \mathrm{s}$ in Figure 5 at $25.64 \mu \mathrm{s}$ ) and its absolute value rapidly decreases as the injection progresses.

To obtain a more global estimate of mass conservation, Table 1 reports the total mass of the liquid phase at discrete times: the linear least-squares fit of these values has a slope of $2.78 \mathrm{~g} / \mathrm{s}$. In the same time interval, the average $\overline{\dot{m}}_{i n j}$ calculated from the simulation is $2.70 \mathrm{~g} / \mathrm{s}$. The estimate average mass flow rate discrepancy is therefore $\overline{\Delta \dot{m}}=0.08 \mathrm{~g} / \mathrm{s}$, corresponding to an error of $+3 \%$ 
with respect to the nominal injection rate. We expect that current work on improving the accuracy of the velocity evaluation at the wall boundary will substantially reduce $\overline{\Delta \dot{m}}$ in future simulations. As a final test, a limited grid-convergence study was carried out by adding a fourth level of refinement up to time $t=11 \mu$ s after the appearance of the jet; and also by carrying out the same simulation with only two refinement levels. This time we consider the mass flow rate at the orifice exit, $\dot{m}_{o}$. The values listed on Table 2 show that $\dot{m}_{o}$ only slightly increases with increasing grid density. The fact that consistently $\dot{m}_{o}<d m / d t$ and that this result does not change substantially with grid refinement will be further discussed in the analysis of the results.

\section{RESULTS AND DISCUSSION}

For both the adiabatic and isothermal wall simulations it is convenient to group our observations in sub-sections, with the understanding that the observed phenomena belong to the same seamless simulation.

\section{INTERNAL FLOW}

The simulation begins with a partially liquid-filled sac. The volume left to the gas inside the injector is $0.065 \mathrm{~mm}^{3}$, or approximately one third of the sac volume (the volume of the cylindrical orifice, of nominal diameter $90 \mu \mathrm{m}$, is much smaller: $0.006 \mathrm{~mm}^{3}$.) The partially filled sac setup is motivated by the observation (via long-distance microscopy and high-contrast display) that gas or vapour 
can be ejected from the orifice in the very early stage of needle unseating: Pickett et al., 2013 reported that gas ejection could be observed before the beginning of injection.

The sequence related to the first part of needle lifting is displayed in the $Z=0$ cross-sections of Figure 6, where time is counted from the activation of the needle. Our first observation is that gas from outside the injector is actually sucked in, from approximately $t=173 \mu$ s after the start of commanded injection (SOI) to $180 \mu \mathrm{s}$. This short-duration inverse flow is due to the receding motion of the needle's tip while the sac is still sealed off from the fuel reservoir: the tip motion is almost imperceptible in this time interval, but the volume displacement is sufficient to cause suction. Later, at time $t=235.5 \mu \mathrm{s}$, the inverted gas jet can be seen to affect the free surface of the fuel.

The obstruction to new liquid entering the cavity begins to be removed a few tens of microseconds later, as the needle begins to lift. This is shown in Figure 6 at $t=315.5 \mu \mathrm{s}$ by the sudden velocity increase at the bottom of the domain. Note that a gap is still not visible in the $Z=0$ cross-section because, as noted before, the lifting of the tip is not axis-symmetrical. The widening of gap is in fact interrupted a few microseconds later because of the wobbling motion of the needle: it is only by $t=330 \mu$ s that the fuel passage completely opens and fuel injection begins. Thus, in the first $300 \mu$ s the needle's motion creates a less than $5 \mu \mathrm{m}$ gap: it is noted that the grid spacing is only 
$3.32 \mu \mathrm{m}$ and that therefore the representation of the flow may be affected in this phase by lack of grid resolution.

Once the needle tip is sufficiently removed from the cap's walls, the liquid begins to fill the sac very rapidly. Two main phenomena are observed. The first is the asymmetric filling of the orifice that leaves a small pocket of trapped gas at the orifice inlet, as shown by Figure 7 . This occurrence can be tracked back to the out-of-axis position of the orifice with respect to the injector. Several smaller bubbles can also be observed. The volume occupied by the residual gas is a small percentage of the initial gas volume, approximately $3 \cdot 10^{-4} \mathrm{~mm}^{3}$, or $0.15 \%$ of the sac volume. The trapped gas is compressed and eventually expelled through the orifice exit.

The second phenomenon is that, as the gas is pushed outside of the orifice, it reaches supersonic conditions that persist until the orifice volume is replaced by fuel (Figure 8). At time $t=337.2 \mu \mathrm{s}$, the gas has reached Mach number 1.2 at the orifice exit and it is expanding outside the injector above Mach two; a few orifice diameters downstream of the injector's exit, pressure has decreased along the axial direction to $0.057 \mathrm{MPa}$. Features of the under-expanded jet and the accompanying barrel shock (with axial compression ratio of 4.5) can be observed after $336 \mu$ s in Figure 9. The left panel corresponds to the $A B$ baseline case of three levels of refinement; the right panel corresponds to four levels of refinement (AB4). The gas flow features are almost identical in the two pictures, with a slightly sharper appearance in the AB4 case. The liquid 
interface instead shows more elongated structures in $A B 4$ that appear as already formed droplets in $A B$.

The barrel shock disappears when the liquid fuel fills the orifice. The fuel emerges from the orifice at $340 \mu \mathrm{s}$. This delay with respect to the SOI depends in part on the amount of residual gas volume inside the sac, and it is reasonable to expect that it could vary between injection cycles. Measurements based on X ray radiography indicate that the jet appears between 305 and $315 \mu \mathrm{s}$, depending on the viewing angle (Kastengren et al., 2014), but the initial amount of gas in the sac in these experiments is unknown. From this point onward it s convenient to eliminate this source of uncertainty and take the time origin to correspond to the appearance of the jet outside of the orifice (ASOI - apparent start of injection).

At the time shown in Figure 10 (approximately $15 \mu$ s after ASOI), the liquid in the sac has reached the inlet pressure value: resistance to the flow is mostly offered by the constriction at the orifice entrance. After passing the gap between the needle and the sac walls, the fuel is actually re-compressed in the sac above the inlet value of $150 \mathrm{MPa}$ before reaching the orifice entrance. There, the pressure abruptly decreases to $80 \mathrm{MPa}$ and inside the orifice it further equilibrates to the external pressure. Correspondingly, the dynamic viscosity in the gap and the sac is $2.4 \mathrm{mPa} \cdot \mathrm{s}$, then decreases to $1.1 \mathrm{mPa} \cdot \mathrm{s}$ at the orifice entry, and eventually reaches a value of $0.64 \mathrm{mPa} \cdot \mathrm{s}$ at the exit of the injector. Past $t=10 \mu$ from ASOI, the fuel density immediately downstream of the 
gap is slightly greater than in the reservoir, as shown in Figure 10 for both cases of adiabatic and isothermal walls; density decreases approaching the sac volume, as the passage width increases, but then it grows again and finally reaches a maximum value of more than $790 \mathrm{~kg} / \mathrm{m}^{3}$. Along the same path, the temperature has variations of opposite sign with respect to density, and reaches $360 \mathrm{~K}$ in the sac. Temperature, pressure and density eventually all decrease in the external jet. The main difference between the isothermal and adiabatic walls cases appears in a slight asymmetry of temperature distribution across the orifice in the AB case, with what appears as a hotter boundary layer on the right-hand side of the orifice's wall in Figure 10. This side is where the flow is more slowed down by the sharper turning angle upstream. In the IB case instead, a well-defined, axis-symmetric thermal boundary layer, of approximately $10-13 \mu \mathrm{m}$ in thickness, is established along the orifice with a temperature of approximately $395 \mathrm{~K}$ around a colder core at $355 \mathrm{~K}$.

\section{RATE OF INJECTION AND MOMENTUM}

We now examine the rate of injection and momentum at the orifice exit. The ROI and ROM from the two test cases are plotted as a function of time in Figure 11 and compared with two sets of measurements (by Sandia and CMT-Motores Térmicos) from the ECN archive (Pickett et al., 2014). Also shown is the reference mass flow from a calibrated model (the "Virtual Injection Rate 
Generator" by CMT, 2015) described in Pyri et al. (2008). The simulation's ROI reaches $\dot{m}_{o}=2.0$ $\mathrm{g} / \mathrm{s}$, or $c_{d}=0.68$, after $25 \mu \mathrm{s}$ from ASOI, whereas at fully unseated conditions the measured value is $2.62 \mathrm{~g} / \mathrm{s}$, corresponding to the discharge coefficient $c_{d}=0.89$. Similarly, the peak ROM is slightly above $1 \mathrm{~N}$ after $25 \mu$ s from ASOI, whereas the measured value is $1.52 \mathrm{~N}$ at fully unseated needle. For the ROI, the mass conservation analysis described in the first part of the paper suggests that part of the discrepancy may be due to the compressibility of the liquid phase: the difference between the average $\dot{m}_{i n j}=2.78 \mathrm{~g} / \mathrm{s}$ at the injector inlet and $\dot{m}_{o}=2.0 \mathrm{~g} / \mathrm{s}$ at the injector exit would therefore correspond to an increase of the liquid density by $\Delta \rho \approx\left(\dot{m}_{i n j}-\right.$ $\left.\dot{m}_{o}\right) \Delta t / V=3.9 \mathrm{~kg} / \mathrm{m}^{3}$ (or $5 \%$ of the ambient density of $\mathrm{n}$-dodecane) per microsecond. This result, obtained by assuming that compression occurs in a volume comparable with the sac's volume, $V$ $=2 \cdot 10^{-4} \mathrm{~cm}^{3}$, is not inconsistent with the process of progressive pressurization of the liquid in the sac. However, regarding the discrepancy in ROM values between experiment and simulation, we recognize that, because of the large Reynolds number inside the orifice, the boundary layer should be much thinner than what is afforded by the available grid resolution. Further investigation of the effect of numerical dissipation in excessively smoothing gradients of velocity, and the implementation of an appropriate wall function to compensate for this effect, are postponed to future work. 


\section{FUEL TEMPERATURE AT THE INJECTOR'S EXIT}

To understand the relation between the fuel's temperature, its equation of state, and frictional effects, it is instructive to start from the limit case of steady, quasi one-dimensional isentropic expansion. Figure 12 displays the iso-contours of $\log _{10} P(\rho, T)$ in the range of density and temperature considered by Caudwell et al. (2004) and illustrates the steep pressure variation of the liquid phase. Also shown in the diagram is the trace in the $\rho-T$ plane of the isentropic expansion from $P=150 \mathrm{MPa}$, with density $787 \mathrm{~kg} / \mathrm{m}^{3}$ and temperature $363 \mathrm{~K}$, to $\mathrm{P}=2 \mathrm{MPa}$, with density $643 \mathrm{~kg} / \mathrm{m}^{3}$ and temperature $340 \mathrm{~K}$. The trajectory is obtained by numerical integration, for sufficiently small decrements $d P$, of the two general relations:

$$
\begin{aligned}
& d \rho=\left(\frac{\partial \rho}{\partial P}\right)_{S} d P=\frac{1}{c^{2}} d P, \\
& d T=\left(\frac{\partial T}{\partial P}\right)_{S} d P=-\frac{T}{c_{p} \rho^{2}}\left(\frac{\partial \rho}{\partial T}\right)_{P} d P,
\end{aligned}
$$

where $S$ is the entropy. The temperature variation in this example is $\Delta T_{\text {exp. }}=-13 \mathrm{~K}$. For comparison, in the case of a perfect gas, Equations (35-36) lead to a close-form solution that is plotted (for $\gamma=1.4$ ) as a thinner line in Figure 12; the end point of that expansion is not visible in the plot because of the much larger density and temperature decrease that is necessary to reach the end pressure of $2 \mathrm{MPa}$.

The cooling of liquid $n$-dodecane in the orifice is contrasted by the enthalpy increase due to friction, particularly when the fuel is forced to pass through the gap between the needle and the 
inner injector wall and later though the injector's orifice. From the compressible Bernoulli equation, the enthalpy variation along a streamline in stationary flow and without heat transfer at the wall can be expressed in terms of the discharge coefficient $c_{d}$ as

$$
\Delta h_{\text {loss }}=\left(1-c_{d}^{2}\right) \frac{\Delta P}{2 \bar{\varrho}} .
$$

The temperature increase can then be approximated from the average value of density and of isobaric heat capacity in the appropriate temperature range. Taking $\bar{\varrho}=761 \mathrm{~kg} / \mathrm{s}$ and $\overline{c_{p}}=2.37$ $\mathrm{kJ} / \mathrm{kg} / \mathrm{K}$ (from the NIST table compiled between $343 \mathrm{~K}$ and $363 \mathrm{~K}$ at $80 \mathrm{MPa}$ ), the estimated temperature increase due to friction is $\Delta T_{f r}=22 \mathrm{~K}$ for $c_{d}=0.68$. With $\Delta T_{\text {exp. }}=-13 \mathrm{~K}$, and assuming superposition of effects, the estimated exit fuel temperature becomes $352 \mathrm{~K}$. Figure 13 displays the time history of the cross-section averaged temperature from the $A B$ and $I B$ simulations. Note that at $25 \mu \mathrm{s}$ after ASOI, the exit temperature reaches a value of $355 \mathrm{~K}$ in the adiabatic wall case, in agreement with this analysis. We expect the temperature to eventually decrease as the needle unseating continues and friction effects are reduced. At the same point in time, the exit temperature reaches the value of $367 \mathrm{~K}$ in the isothermal wall case. While the temperature difference between IB and $A B$ cases cannot be neglected, the cross-section averaged exit fuel density is almost identical: it is $711 \mathrm{~kg} / \mathrm{m}^{3}$ at the exit of the injector for the adiabatic case and $706 \mathrm{~kg} / \mathrm{m} 3$ for the isothermal case, starting from the value $790 \mathrm{~kg} / \mathrm{m}^{3}$ upstream of the needle gap. 


\section{EXTERNAL FLOW}

Because of the large injection pressure, the velocity of the fuel at the exit of the orifice reaches $600 \mathrm{~m} / \mathrm{s}$ - a supersonic velocity in the gas phase, but less than half the speed of sound for dodecane. Not surprisingly, because of the asymmetry that was purposely introduced in the injector model, the shape of the tip of the jet (see Figure 14) is far from the regular shape that is often assumed as the initial condition of injection simulations (say, a cylinder terminated by a half sphere). Eventually however, the peeling of ligaments from the jet core in the interaction with the gas flow causes a tip shape similar to a mushroom head; see Figure 14, which renders the jet in a reference frame moving with its tip.

In this phase of injection, a detached bow shock forms ahead of the jet tip, as shown in Figure 15. This flow feature is consistent with the observation by Kook and Pickett that (2008) that, at sufficiently low chamber temperature, the high-density ambient gas quickly decelerates the liquid jet causing a lead shock wave to detach from the spray. Interestingly, in the wake of the leading bow shock, stronger shocks can be localized in Figure 15 close to the liquid fragments.

A more precise idea of the jet topology is provided by scanning its cross-sections. Figure 16 displays cross-sections taken $24.2 \mu$ s after ASOI for the adiabatic case: they rapidly transition from a relatively regular, but jagged, circular shape to a more elongated and irregular shape before starting to disintegrate at approximately $2.5 \mathrm{~mm}$ from the orifice exit. The sample-averaged 
volume fraction profiles of the liquid phase derived from projection of radiography data by Pickett et al. (2014) similarly fall below unity between 1.2 and $2.4 \mathrm{~mm}$ from the orifice exit.

In Figure 17 the penetration of the jet emerging from the injector is directly compared to x-radiographic measurements by ANL (data downloaded from the ECN archive) obtained at cold gas flow conditions. The ANL symbols in Figure 17 correspond to the ensemble average of several fuel injection cycles and account for the varying time origin of the ASOI, which we assume to be the same as in the simulations. Recalling that the liquid surface is available from the interface-capturing algorithm, the jet penetration can be defined independently from a density threshold value as the distance from the injector's orifice to the furthest point of the uninterrupted jet. The abrupt change of length of the computed liquid core shown in Figure 17 corresponds to the jet breaking up (the fact that the jet length is sampled at relatively spaced intervals partially masks this abrupt variation). After jet breakup, one or more large blobs of liquid are formed and then rapidly disintegrate. Overall, the trajectory of the jet tip and the penetration prediction appear in good agreement with the ANL data. Note that there is not a substantial difference in the early trajectory of the jet tip between the adiabatic and isothermal wall simulations, as the exit density and velocity are similar in both cases. Where a difference is present, it is in the tendency of the IB case to break more often and at earlier locations. However, several simulation instances, not available at this point, would be necessary to approach an ensemble-averaged jet breakup length 
for a more consistent comparison with the data: currently we cannot quantify what would be the variation amongst different realizations obtained with the same wall boundary conditions. With this caveat, the jet breakup point is identified to be $6 \mathrm{~mm}$ from the orifice exit in the IB case and 7 $\mathrm{mm}$ in the $\mathrm{AB}$ case. Consistent with this trend with temperature, at a much higher gas temperature (900 K), the jet tip position at $\mathrm{t}=25 \mu \mathrm{s}$ after ASOI was $5.5 \mathrm{~mm}$ according to a combination of long-distance microscopy, Mie-scatter imaging with side illumination, and diffused back illumination data by Jung et al. (2015).

With at least a partially validated methodology for calculating spray atomization, we can examine the distribution of liquid drops immediately after formation, for instance to characterize what their size is and how close they are to the spherical shape. For a given snapshot of the solution, the zero iso-surface of the liquid-gas level set is extracted in a post-processing stage and reduced to a tessellation made of triangular or quadrilateral faces. A simple procedure then separates from each other structures that do not have nodes in common, generating polyhedra that correspond each to a distinct liquid shape. The number of faces of a polyhedron may vary from several thousands to a few tens. From them, surface and volume are then calculated. The volume calculation is carried out by subdividing each polyhedron in tetrahedra or pyramids, whose base is one the faces and whose vertex is a reference point (the "center of mass" of all the nodes in the shape, for instance). This procedure still works for non-convex shapes where the reference point 
is outside of the shape, as long as the volumes in the decomposition are summed with sign: the volume value is positive only if the normal vector from the face, pointing toward the interior of the shape, also points toward the reference point. Finally, the diameter of equivalent spheres, in either volume $D_{v}$ or surface $D_{s}$, can be evaluated.

To assess the convergence of the resulting spray distribution, the snapshot at $6 \mu$ srom ASOI of the four-level refinement $A B 4$ simulation was processed and compared with the distribution from $A B$ at the same point in time. Note that in both snapshots the incipient spray appears to be mostly formed by ligaments and that the equivalent diameter is insufficient to describe their shape. With this caveat, the two distribution plots for $D_{v}$ are shown in Figure 18. In comparing the two diagrams, we assume that droplets with equivalent diameter below $2 \Delta \mathrm{x}$ are not sufficiently supported by the grid resolution and need to be discarded (but methods exist to transfer their representation to Lagrangian particles; see, for instance, Herrmann, 2010). With 145 remaining samples for $\Delta \mathrm{x}=3.32 \mu \mathrm{m}$ and 269 for $\Delta \mathrm{x}=1.66 \mu \mathrm{m}$, we see that the $A B$ distribution is slightly skewed toward larger droplets (each histogram is normalized by the total number of the samples). Comparing the two simulation snapshots reveals that some intact ligaments in the AB4 simulation appear to have already undergone breakup in $A B$, similarly to what shown in Figure 9. The spurious breakup of a liquid surface is a well-known signature of insufficient grid resolution: a previous study by the authors (Arienti et al., 2013) showed that an interface-capturing technique 
similar to the one used here eventually yielded to a converging spray size distribution, which in turn approached the measured distribution, under sufficient grid refinement. Thus, for the current study we can assume that only the intermediate-to-large size droplet population is converged, while acknowledging that the majority of droplets observed in the actual experiment is actually left out from the baseline simulation.

To compare the overall spray distribution from the adiabatic and isothermal wall conditions at a later time in the injection process, Figure 19 displays the $D_{v}$-based size distribution ( $A B$, red line, at $\mathrm{t}=21.2 \mu \mathrm{s}$ and IB, black line, at $\mathrm{t}=19.6 \mu \mathrm{s}$. There are 20,495 samples (of which 9044 between $2 \Delta x$ and $90 \mu s$ ) in the first case, and 38,740 samples (of which 16997 between $2 \Delta x$ and $90 \mu \mathrm{s}$ ) in the second case: large liquid structures, detached from the jet core but not yet fully atomized, do not show in the scale of the plots. The Sauter Mean Diameter, SMD $=\sum_{i}^{N} D_{V}^{3} / \sum_{i}^{N} D_{V}^{2}$, conditioned to the range $6.64 \mu \mathrm{m}<\mathrm{D}_{v}<90 \mu \mathrm{m}$ is $\mathrm{SMD}=17.2 \mu \mathrm{m}$ at $\mathrm{t}=19.6 \mu \mathrm{s}$ in the IB case; but $\mathrm{SMD}=15.5$ $\mu \mathrm{m}$ (with $60 \%$ more droplets analyzed) eight microseconds later. Similarly, for the AB case, SMD $=28.9 \mu \mathrm{m}$ at $\mathrm{t}=21.2 \mu \mathrm{s}$ and $\mathrm{SMD}=15.7 \mu \mathrm{m}$ at $\mathrm{t}=25.6 \mu \mathrm{s}$.

The confirmation of these results by experimental data is at the moment limited, also because few direct measurements of droplet size distribution are available for spray A. The existing measurements show some dispersion. For a single-orifice Diesel injector similar to Spray A, Powell et al. (2013) used Ultra-Small Angle X-ray Scattering (USA-XS) under the assumption of 
spherical, randomly oriented particles, to evaluate the Sauter Mean Diameter (SMD) as a function of axial distance: they found $\mathrm{SMD} \approx 3.2 \mu \mathrm{m}$ at a location $4 \mathrm{~mm}$ downstream of the orifice exit. At $900 \mathrm{~K}$ gas temperature, SMD was even smaller, less than $1 \mu \mathrm{m}$, according to preliminary results presented by the same group at the fourth ECN workshop in 2015. An alternative to USA-XS is the microscopic imaging technique by Crua (Crua et al., 2012). In the optically thin region at the edge of the spray cone where Crua's technique was used, the droplets' SMD was much larger, in the range between 5 and $8 \mu \mathrm{m}$ according to very preliminary results also presented at the fourth ECN workshop in 2015. Currently these measurements cannot be extended to the core of the spray where droplets could be much smaller.

From Figure 19, we see that the two droplet diameter distributions show some dependence on the wall boundary conditions: the $\mathrm{T}_{\mathrm{w}}=383 \mathrm{~K}$ case tends to produce more droplets in the smaller-diameter population (say, for $D_{v}<12 \mu \mathrm{m}$ ) than the adiabatic case. We attribute this behavior to the higher temperature of the liquid thermal layer in the orifice in the IB case, which translates into lower values of the local surface tension coefficient and therefore into increased atomization. This same argument applies to the shorter jet core length observed earlier. However, this difference is quite subtle and caution should be exerted in interpreting differences between the two individual realizations of Figure 19.

We conclude this Section with the assessment of whether drops formed from primary atomization 
can be considered spherical so close to the jet core. The issue can be examined by looking at the histogram of the ratio $D_{v} / D_{s}$, conditioned to the same size filter as before. With the understanding that the ratio $D_{v} / D_{s}=1$ corresponds to a perfectly spherical drop, Figure 20 shows that more than a third of the droplets falls into the narrow bucket between 0.94 and 0.98 ; a third more belongs to the range between 0.86 to 0.94 ; but a remaining third is such that $D_{v} / D_{s}<0.86$. This last tier points to the many liquid structures forming from the jet that are topologically complex and tend to fragment further (in the process called secondary atomization). We also see from the normalized diagram that drops from the $A B$ injection maintain a deformed spherical shape in slightly greater proportion than the drops form the IB injection. With caution, we attribute this effect to the temperature dependence of the surface tension coefficient: drops with stronger surface tension equilibrate to a spherical shape faster.

\section{CONCLUSIONS}

We have applied a high-fidelity multiphase computational method to the study of the early transient of needle unseating. The simulations demonstrate the ease of the proposed methodology for dealing with moving boundaries and for including compressibility effects in the description of the liquid phase. Two limit cases are considered, one with adiabatic wall boundaries and the second where the wall temperature is set to a constant value. In the discussion section, we have commented on the following flow features: 
- At the onset of needle lifting, a small amount of gas is ingested in the sac due to the interference between needle tip and injector walls: the receding tip is freeing volume inside the sac, but it is not displaced enough to open a passage for the fuel. The actual amount of gas inside the injector appears to depend on the geometrical fit of the needle's tip inside the injector and on the residual amount of fuel left from the previous injection cycle. We speculate that these dependencies may contribute to cycle-to-cycle variations of spray characteristics and perhaps even to performance differences amongst injectors of the same model. These hypotheses need to be tested in future work.

- As soon as a gap opens between the needle tip and the internal wall of the injector, most of the gas contained in the sac is ejected, forming an under-expanded jet outside of the orifice. The fuel jet then rapidly replaces the gas jet. The first droplets actually form inside the orifice in this early phase.

- The calculated rate of injection at the injector's orifice from the simulation is $25 \%$ smaller than the value measured in the fully open configuration. The fact that the simulated time is not sufficient to reach the fully open configuration might partially explain this discrepancy. Moreover, by adding one level of refinement the ROI could increase by $5-10 \%$. However, we have verified that the liquid mass rate that enters the domain is consistently larger than the rate at the orifice, 
and this seems to point to a continuing compression of the fuel inside the sac. This hypothesis needs to be tested with longer simulation times.

- Similarly, the calculated rate of momentum at the injector's office is substantially smaller than the measured value. We attribute this result to the fact that the Reynolds number that can actually be resolved in the simulation is quite small and to the excessively thick boundary layer in the orifice. The boundary layer thickness at fully open condition is estimated to be of the order of a micrometer or less in the orifice. As the embedded boundary method does not allow for special grid spacing near the walls, the implementation of an appropriate wall function is planned for future work.

- The fuel temperature at the orifice exit during the opening transient remains within 15 Kelvin from the prescribed reservoir temperature with adiabatic wall conditions. This increase is comparable with our estimate of the effects of liquid phase expansion due to the pressure differential and of enthalpy increase due to viscosity. As the needle becomes fully unseated, the remaining viscous effects concentrate at the orifice inlet and the exit temperature tends to reach a constant value. With isothermal wall conditions, $T_{w}=383 \mathrm{~K}$, the exit temperature reaches $368 \mathrm{~K}$ and then it seems to stabilize around that value.

- The exit fuel density decreases from $790 \mathrm{~kg} / \mathrm{m}^{3}$ upstream of the needle gap to $711 \mathrm{~kg} / \mathrm{m}^{3}$ at the exit of the injector for the adiabatic case, and to $706 \mathrm{~kg} / \mathrm{m}^{3}$ for the isothermal case. 
Correspondingly to these almost identical density values, the early trajectory of the jet tips are also quite similar.

- As the liquid jet exits the orifice faster than the speed of sound in the gas phase, shock formation is expected. However, due to the non-uniform filling process of the sac during needle unseating, the liquid jet exits the injector in a partially fragmented shape form and does not apply a uniform compression to the external gas. The leading bow shock is detached from the tip because the jet is rapidly decelerated by the gas. In its wake, stronger shocks can be identified just ahead of the most prominent liquid structures.

- The penetration of the uninterrupted core of the jet is consistent with sample-averaged X-radiography measurements of the trajectory of the tip at cold gas conditions and is similar for both wall boundary conditions. This last result is attributed to the dependence of the tip trajectory on exit liquid density, which is almost the same in the two cases.

- Conversely, the jet break-up length, which we identify from the sudden shortening of the uninterrupted core, occurs within $7 \mathrm{~mm}$ from the orifice for the adiabatic wall case and within $6 \mathrm{~mm}$ for the isothermal case. This difference is attributed to the built-in dependence of the surface tension coefficient on temperature and it is so far the main visible effect attributable to the two wall conditions. 
- In the process of jet breakup, a few large chunks of liquid (larger than $d_{0}$ ) get separated from the core, while a broad variety of smaller drops is detected. We calculated spray size distributions based on the drop population detected at a given instant with equivalent diameter in the range from 6.64 to $90 \mu \mathrm{m}$ (drops below twice the grid spacing may be not be sufficiently well captured). From the distributions we see a trend toward smaller droplets from the adiabatic to the isothermal case that we attribute to the exit temperature difference between the two simulations. However, this difference is very small and could also be attributed to intrinsic variability in the two realizations.

- Drops in the same intermediate-to-large equivalent diameter range were also examined with respect to their shape, taken as an indicator of whether they will further fragment before equilibrating with the surrounding gas flow: approximately two thirds of both sample populations were found to possess a shape sufficiently close to spherical, while the remaining third has a more elongated shape (ligaments) and could undergo secondary breakup.

Future work will require additional analysis on a longer fraction of the injection cycle and with more grid refinement: but the continuation of the present simulations has to contend with the fact that the process of injection and spray formation is a formidably "stiff" problem, where the stable simulation time step is six orders of magnitude smaller than the injection period. The implementation of wall models suited to regions where cavitation and high Reynolds number 
turbulence are present is the main priority to continue this investigation on the effects of internal flow on atomization by direct fuel injection.

\section{ACKNOWLEDGMENTS}

Support by Sandia National Laboratories' LDRD (Laboratory Directed Research and

Development) is gratefully acknowledged. Sandia National Laboratories is a multi-program laboratory managed and operated by Sandia Corporation, a wholly owned subsidiary of Lockheed Martin Corporation, for the U.S. Department of Energy's National Nuclear Security Administration under contract DE-AC04-94AL85000. 


\begin{tabular}{|l|l|}
\hline Time $[\mathrm{ms}]$ & Mass $[\mathrm{mg}]$ \\
\hline 0.350308877 & 0.386239 \\
\hline 0.353472345 & 0.394559 \\
\hline 0.355085879 & 0.399395 \\
\hline 0.356358432 & 0.402816 \\
\hline 0.359861332 & 0.412795 \\
\hline 0.361368221 & 0.416869 \\
\hline 0.363737442 & 0.423338 \\
\hline
\end{tabular}

Table 1. Sequence of total liquid mass calculated from the entire computational domain. 


\begin{tabular}{|l|c|c|c|}
\hline $\begin{array}{l}\text { Refinement } \\
\text { level }\end{array}$ & 2 & 3 & 4 \\
\hline$\Delta x[\mu \mathrm{m}]$ & 6.64 & 3.32 & 1.66 \\
\hline$\dot{m}_{o}[\mathrm{~g} / \mathrm{s}]$ & 1.54 & 1.75 & 1.82 \\
\hline$\dot{q}_{o}[\mathrm{~N}]$ & 0.74 & 0.79 & 0.80 \\
\hline
\end{tabular}

Table 2. Mass and momentum flow rate at $11 \mu$ s after ASOI from simulations with decreasing grid spacing. 


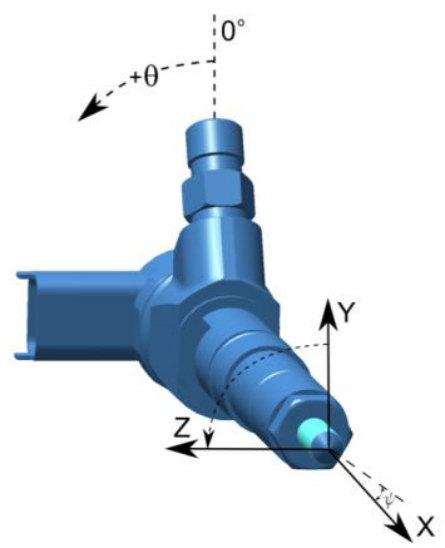

Figure 1. Spray A and reference coordinates. 


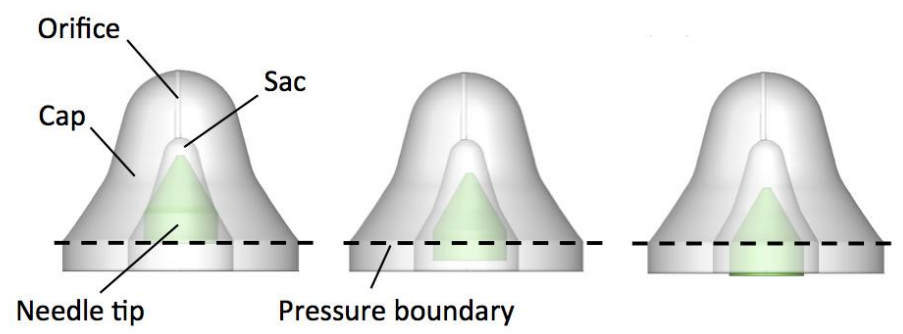

(a)

(b)

(c)

Figure 2. Sequence illustrating the relative motion of the needle tip with respect to the cap.

The dashed line is the trace of the boundary plane of the computational domain. 


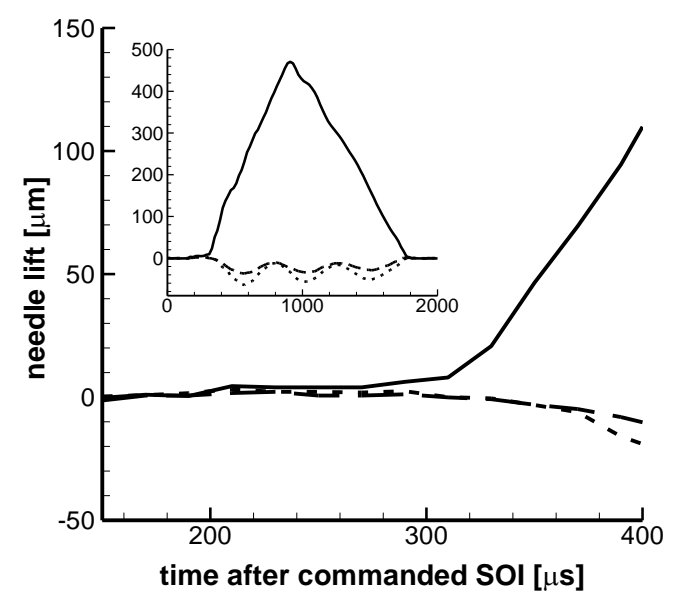

Figure 3. Trajectory of the needle with respect to the cap for the $\mathrm{X}-\mathrm{Y}$ - and Z-components (continuous, dashed and dotted line, respectively). The insert shows the complete trajectory of the needle, in the same units. 


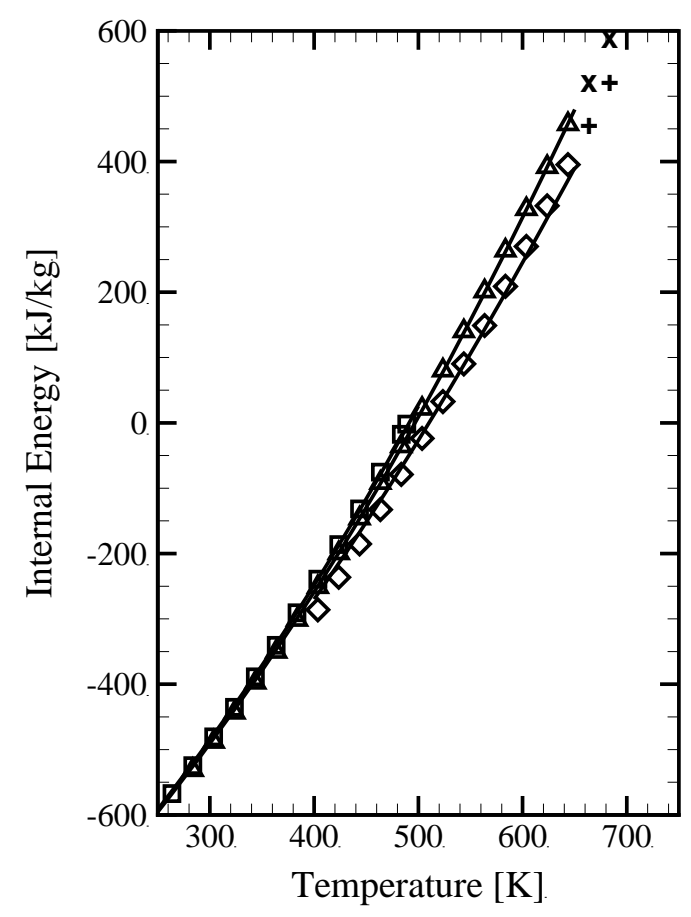

Figure 4. Internal energy of $n$-dodecane at constant pressure $P=0.1,20$ and $140 \mathrm{MPa}$ (lines in order of decreasing slope) compared to NIST data (square, triangle and lozenge symbols, respectively). The $\mathrm{x}$ and + symbols refer to the supercritical state at 20 and $140 \mathrm{MPa}$. 


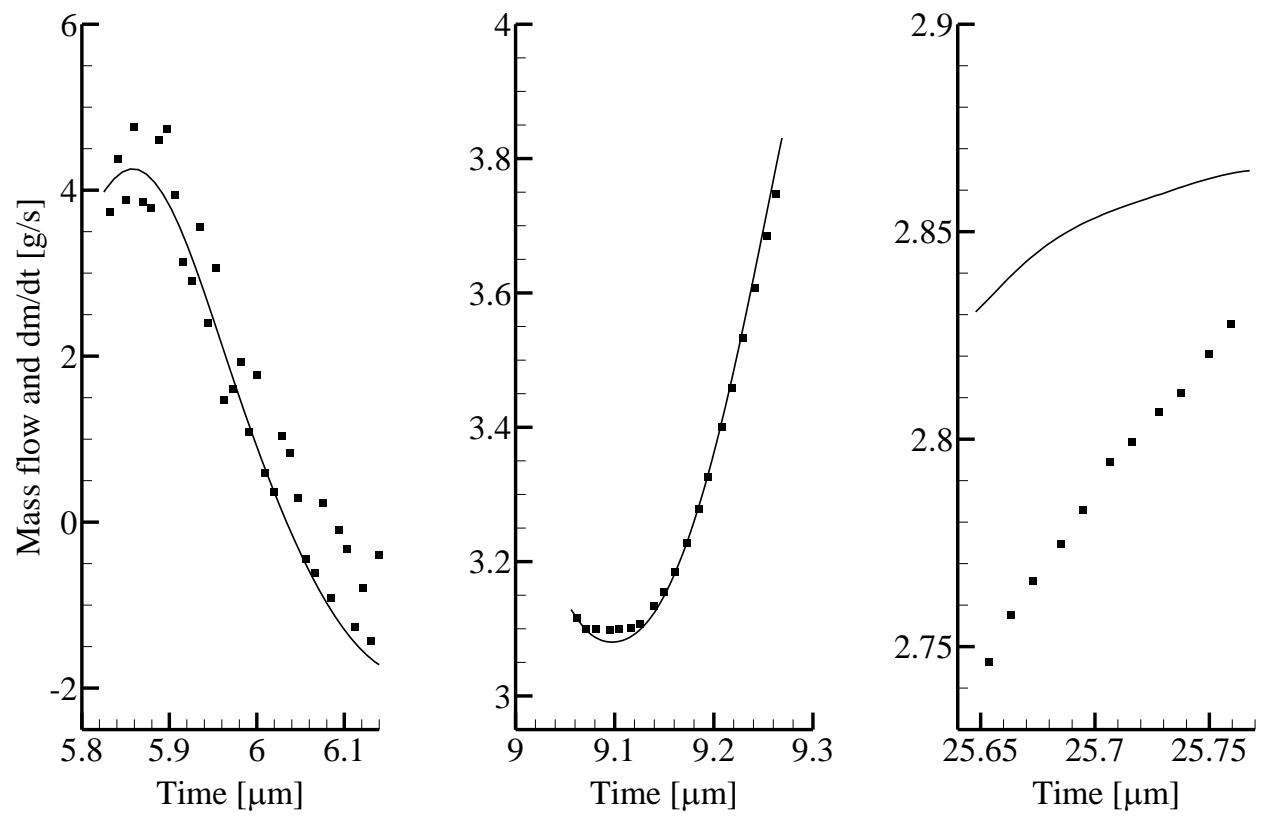

Figure 5. Mass flow rate through the inlet (lines) compared to the derivative of the total mass of the liquid fuel (symbols) at three different points in the injection sequence. The time is calculated from the appearance of the jet outside of the injector. 

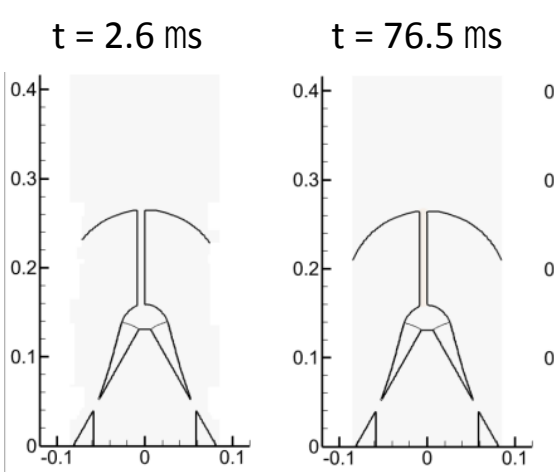

$t=158.8 \mathrm{~s}$
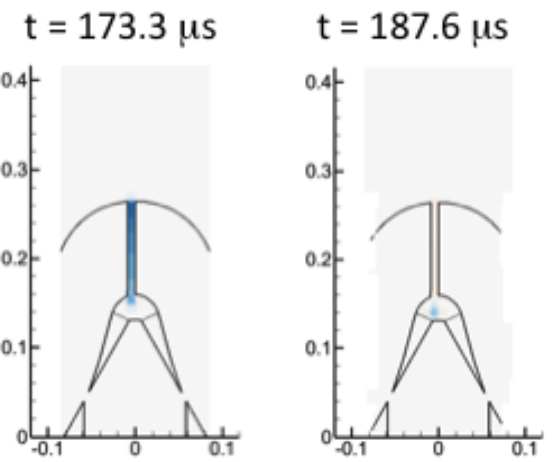

$\mathrm{t}=235.5 \mu \mathrm{s}$
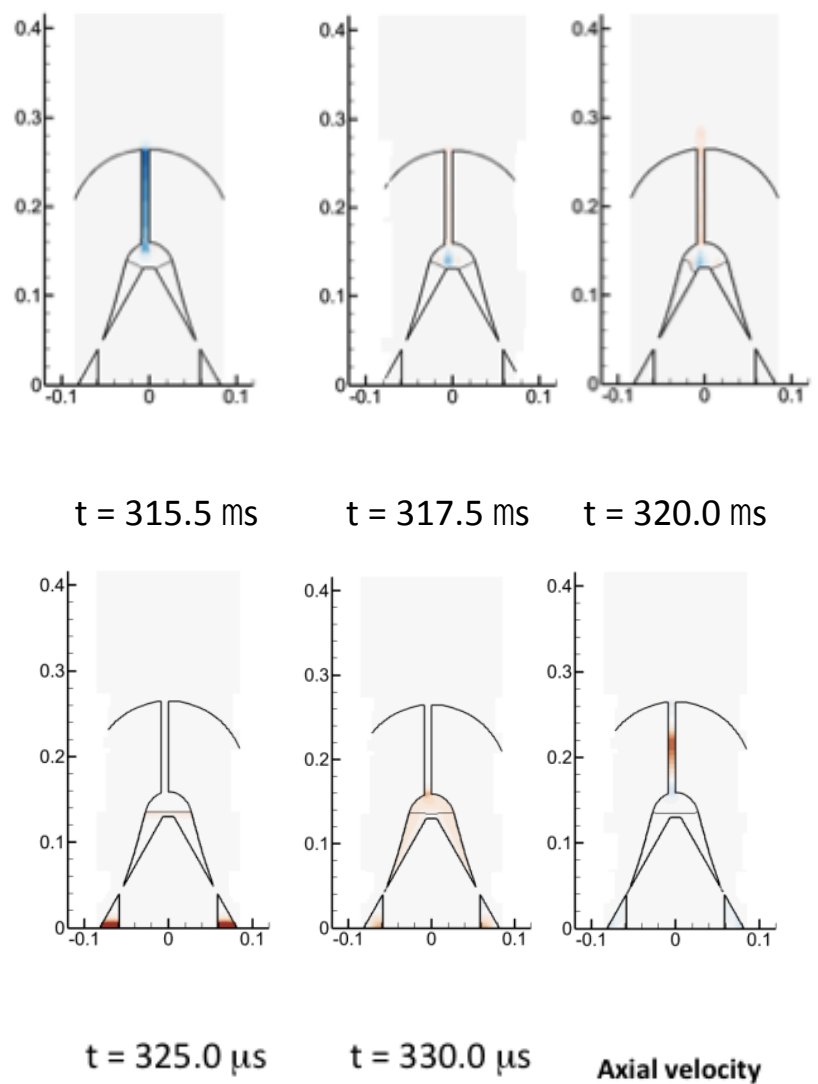

Axial velocity
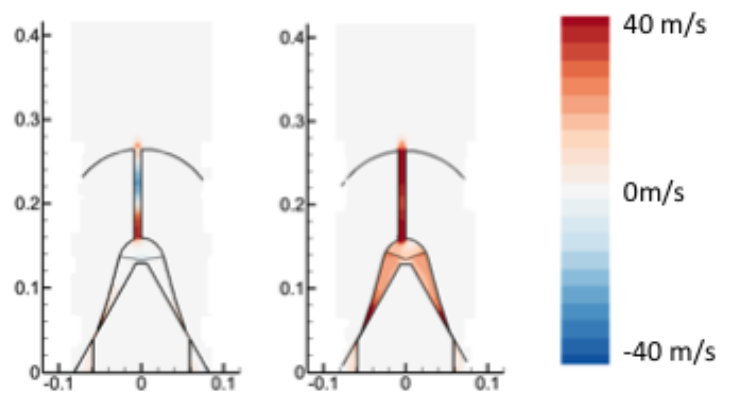
Figure 6. Axial velocity of the gas and liquid phase in the early opening transient as it appears

in the $Z=0$ cross-section of the domain. The thicker continuous line corresponds to the boundaries of the needle and the orifice, while the thinner line represents the free surface of the liquid. 


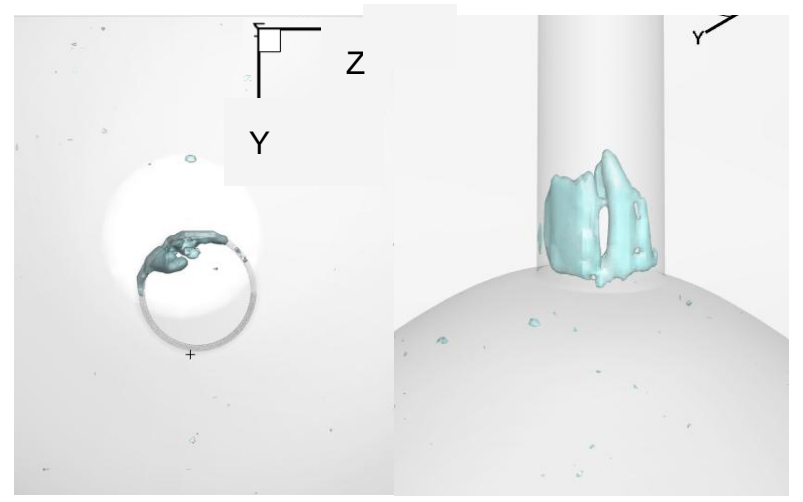

Figure 7. Front and side view of gas bubble at $339 \mu$ s. 

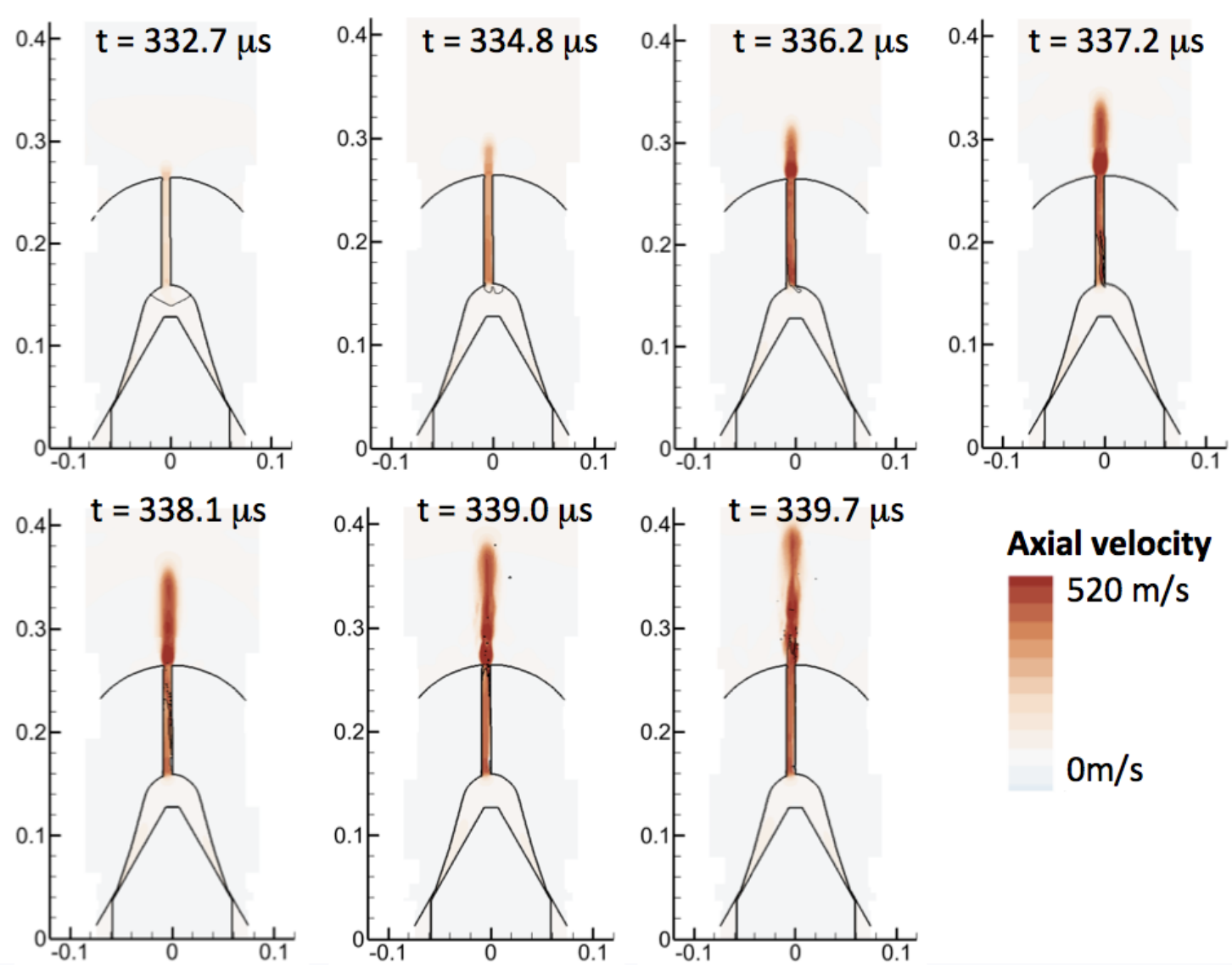

\section{Axial velocity}

$520 \mathrm{~m} / \mathrm{s}$

$0 \mathrm{~m} / \mathrm{s}$

Figure 8. This is a continuation of Figure 6 and follows the same drawing conventions. Note

the increase of axial velocity by a factor of 10 with respect to the previous injection interval. The last frame shows the appearance of the jet outside of the injector. 


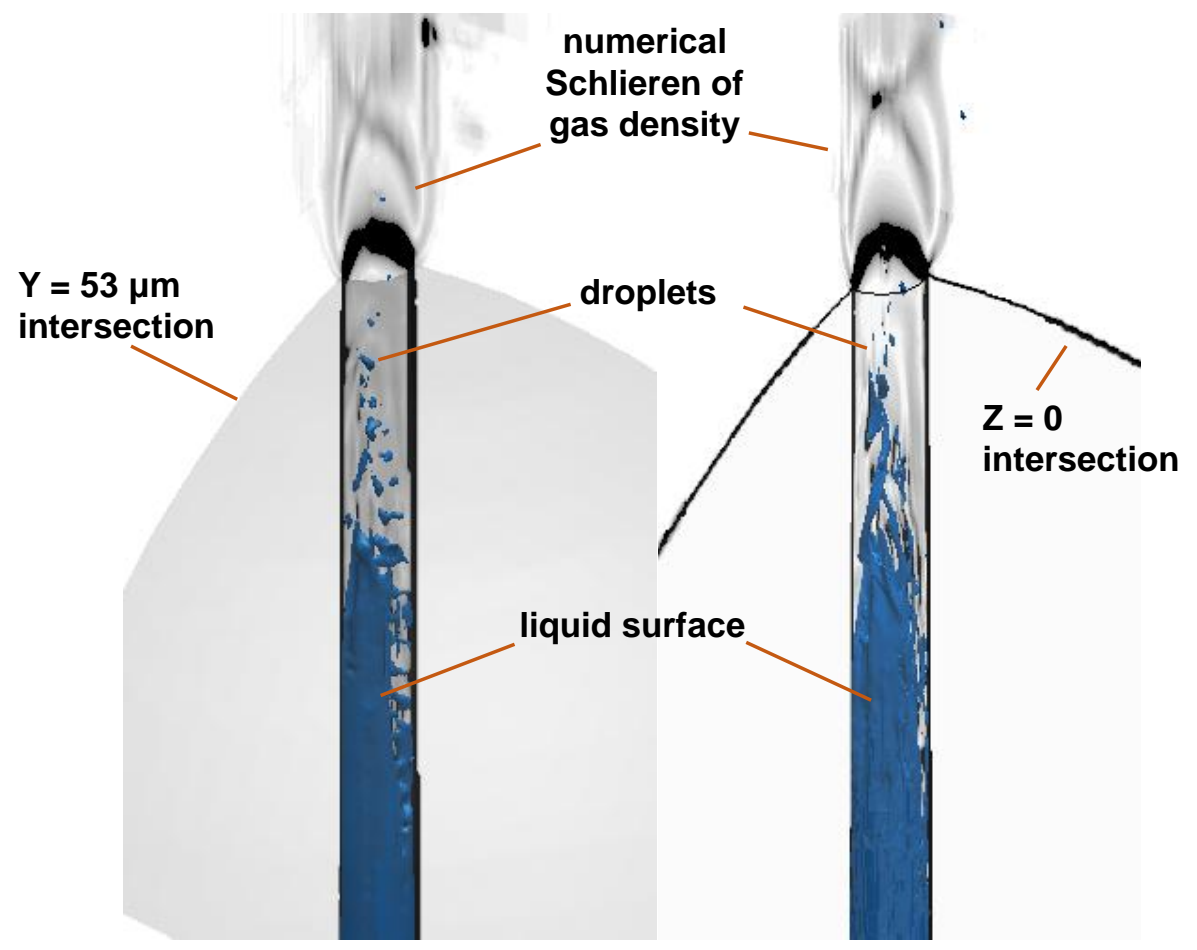

Figure 9. Detail of under-expanded gas jet (shown by numerical Schlieren) at $t=337.2 \mu$ s on two orthogonal slices crossing at the axis of the orifice. Also shown is the volume rendering of the liquid surface at the same time. Left: with three levels of refinement $(\Delta x=3.32 \mu \mathrm{m})$; Right: with four levels refinement $(\Delta x=1.66 \mu \mathrm{m})$. 

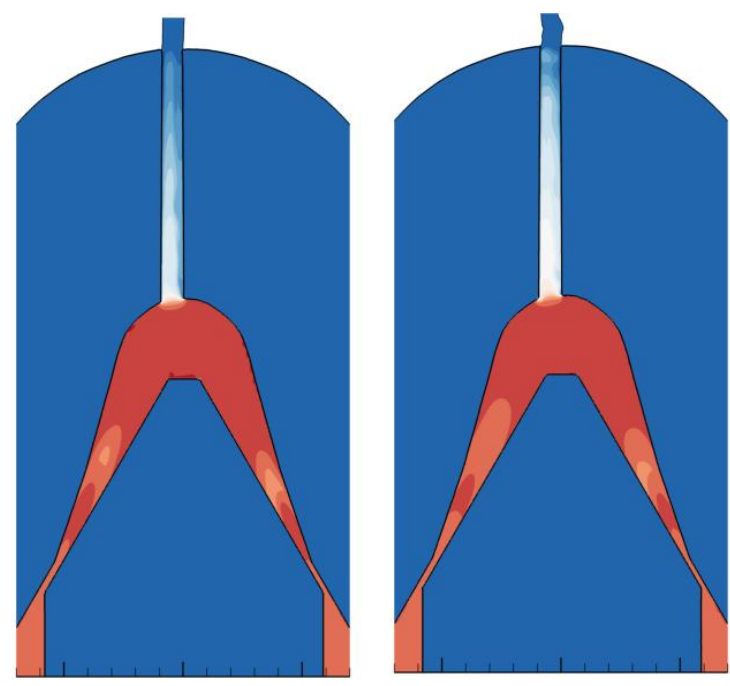

$\begin{array}{llllll}1 & & & & & \end{array}$

Density $\left[\mathrm{kg} / \mathrm{m}^{3}\right]$

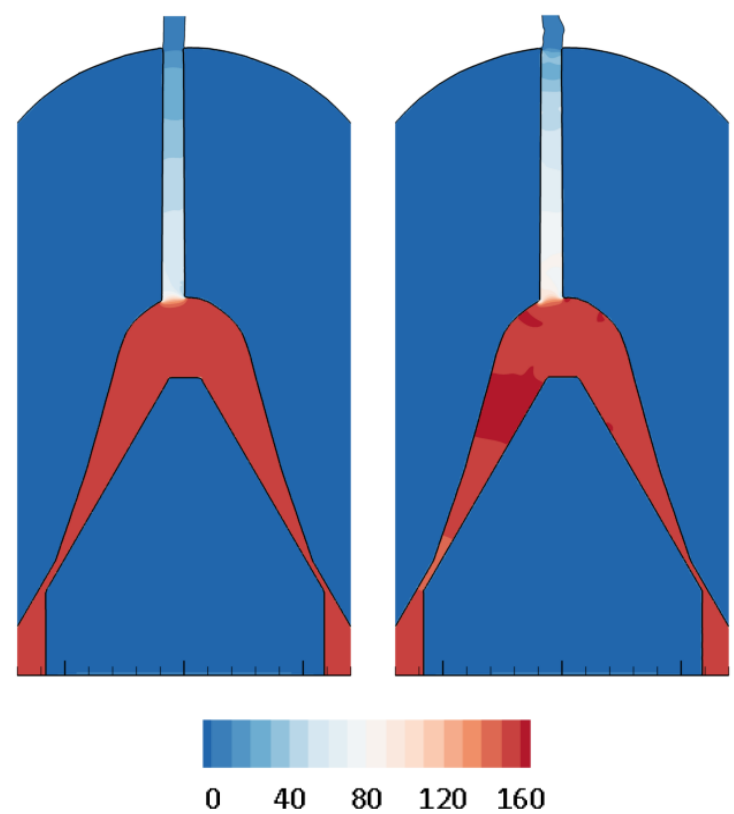

Pressure [MPa] 


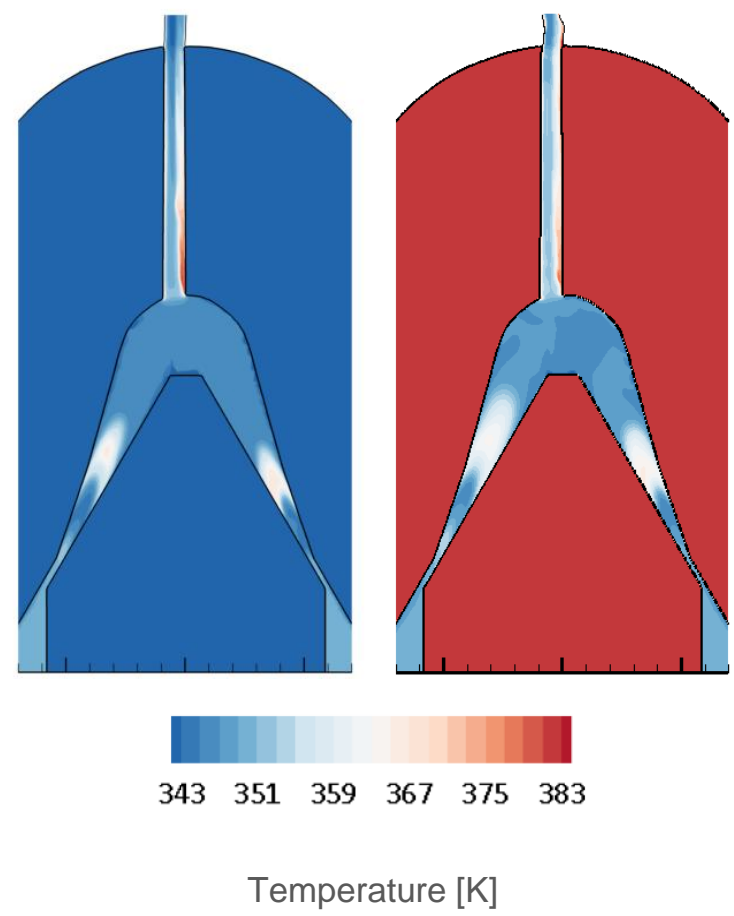

Figure 10. Distribution of density, pressure and temperature at the $Z=0$ cross-section (gas phase not shown). Left: adiabatic injector walls $(A B)$ at $t=354 \mu$ s. Right: injector walls at fixed $T_{w}$ $=383 \mathrm{~K}(\mathrm{IB})$ at $\mathrm{t}=352 \mu \mathrm{s}$. 

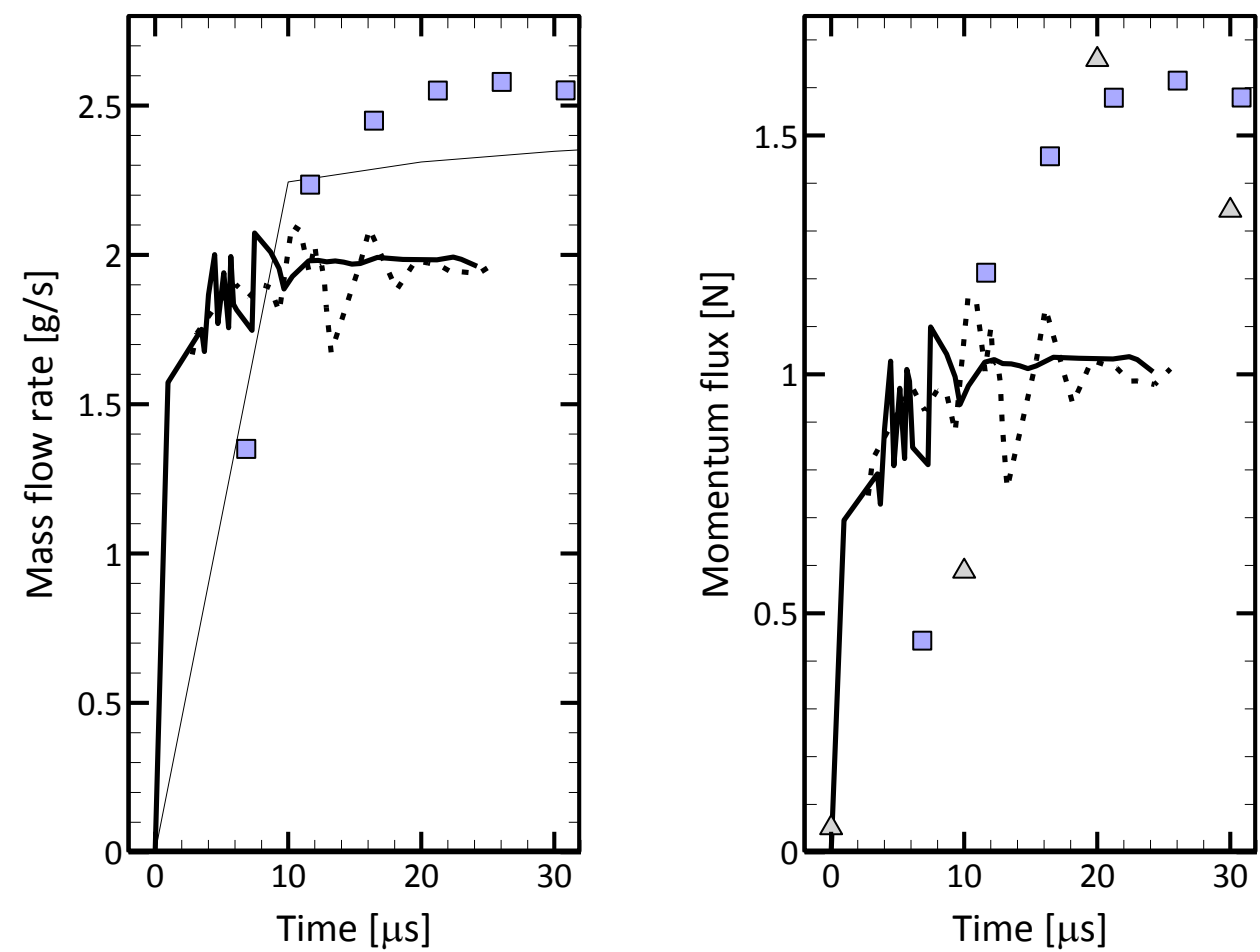

Figure 11. Left panel: rate of injection from the adiabatic-wall simulation (continuous line) and the isothermal wall simulation (dashed line) compared with measurements from Sandia (squares). The thin line is the output of a ROI model obtained by calibration from a group of similar injection experiments. Right panel: rate of momentum, same legend. The triangular symbols are ROM measurements from CMT. 


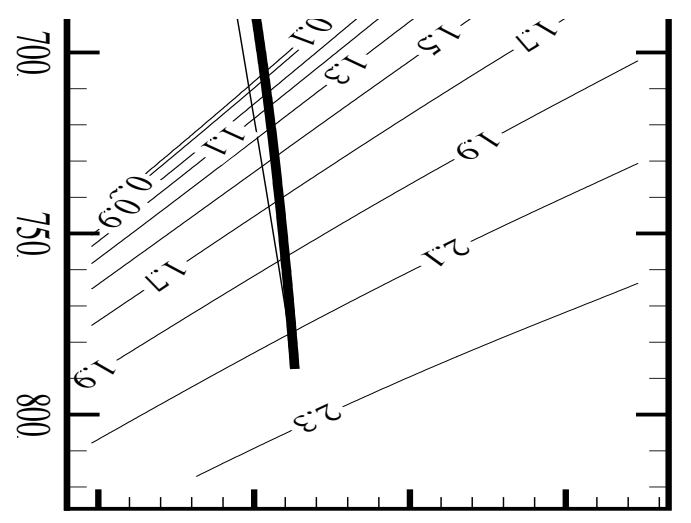

Figure 12. Logarithm (base 10) of pressure (in MPa) calculated as a function of temperature and density from the EOS of $n$-dodecane. The thick line is the trace of the isoentropic expansion from $150 \mathrm{MPa}$ to $2 \mathrm{MPa}$. The thin line is part of the the isoentropic expansion for the same pressure drop but calculated for a perfect gas with $\mathrm{y}=1.4$. 


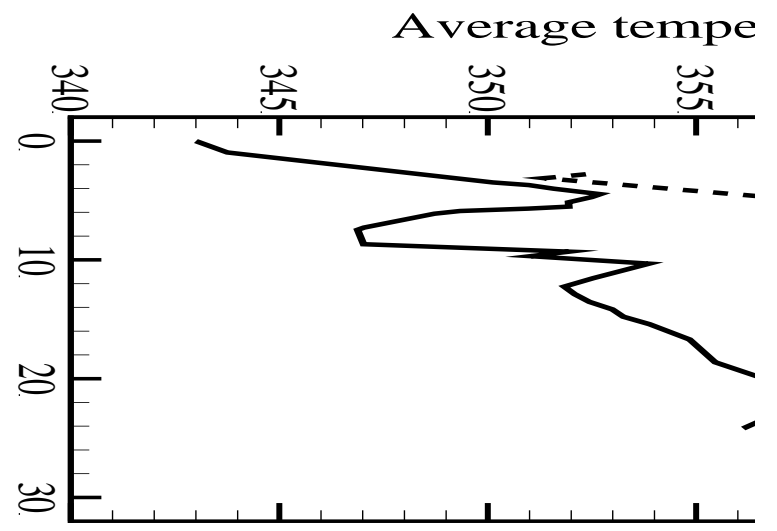

Figure 13. History of exit temperature averaged over the cross-section of the orifice.

Continuous line: adiabatic wall simulation. Dashed line: isothermal wall simulation. 

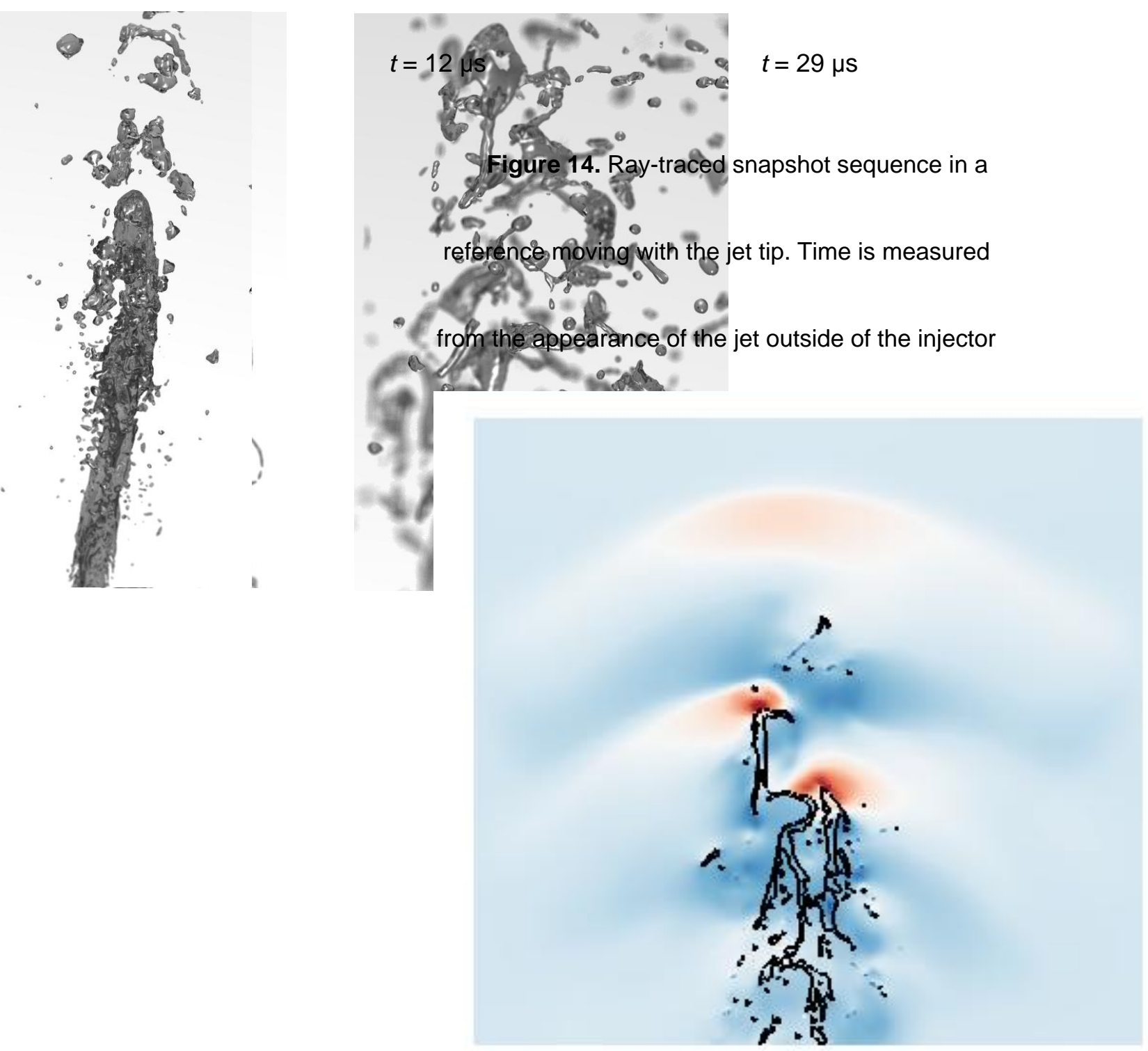

Figure 15. Snapshot $(Z=0$ cross-section) of pressure field from 1.2 to $3.4 \mathrm{MPa}$ at $\mathrm{t}=12.9 \mu \mathrm{s}$ from ASOI. Also shown is the trace of the liquid surface. At this time the leading bow shock is located at $5.1 \mathrm{~mm}$ from the orifice exit. 


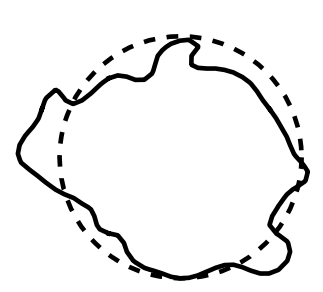

(a)
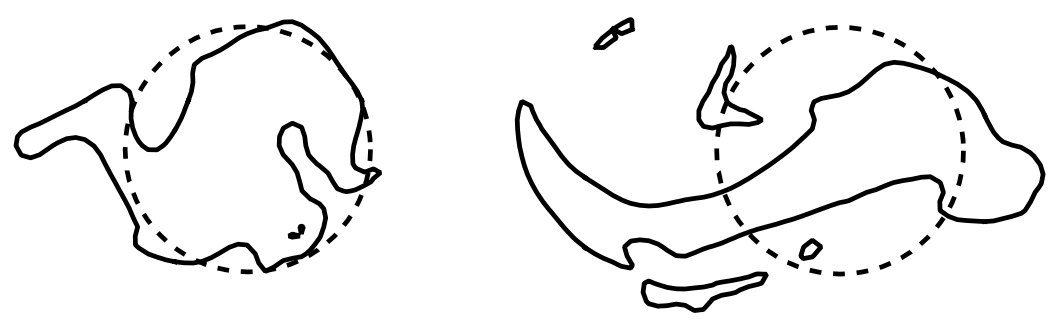

(b)

(c)

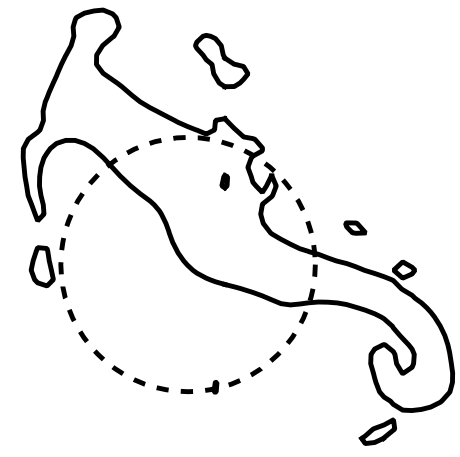

(d)

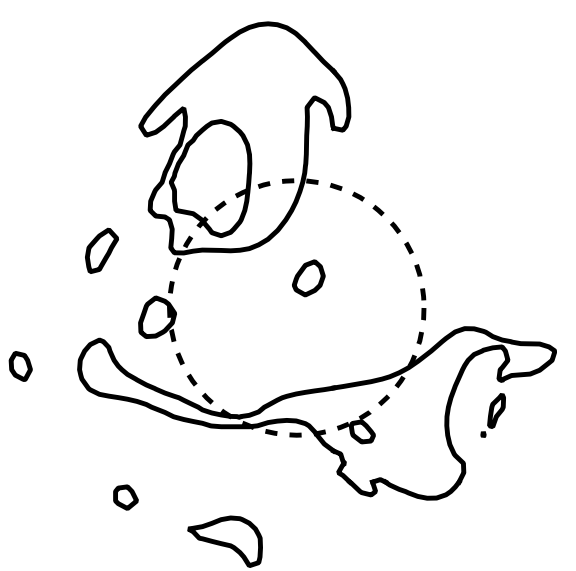

(e)

Figure 16. Scan at $t=24.2 \mu$ s of the liquid phase cross-sections in the adiabatic wall case.

(a): $X=0.37 \mathrm{~mm}$; (b) $X=0.97 \mathrm{~mm}$; (c): $X=1.77 \mathrm{~mm}$; (d) $X=2.57 \mathrm{~mm}$; (e) $X=2.77 \mathrm{~mm}$. The circular dashed line indicates the trace of the orifice's exit in the $\mathrm{Y}-\mathrm{Z}$ plane. 


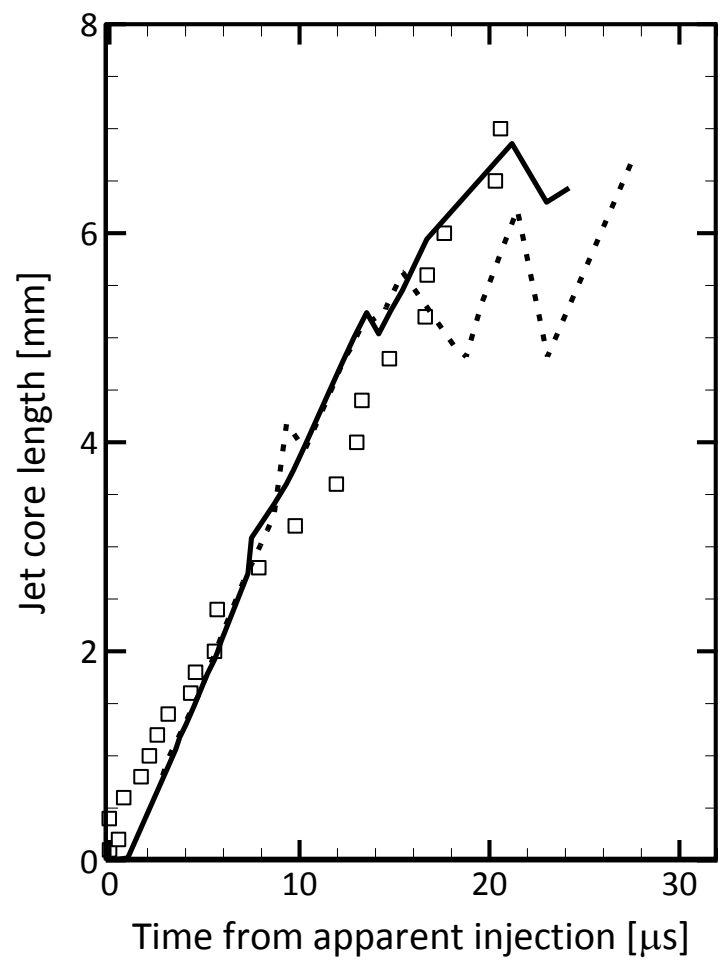

Figure 17. Fuel jet penetration from the adiabatic (continuous line) and isothermal (dashed line) wall simulations compared to sample-averaged measurements from ANL (square symbols). 

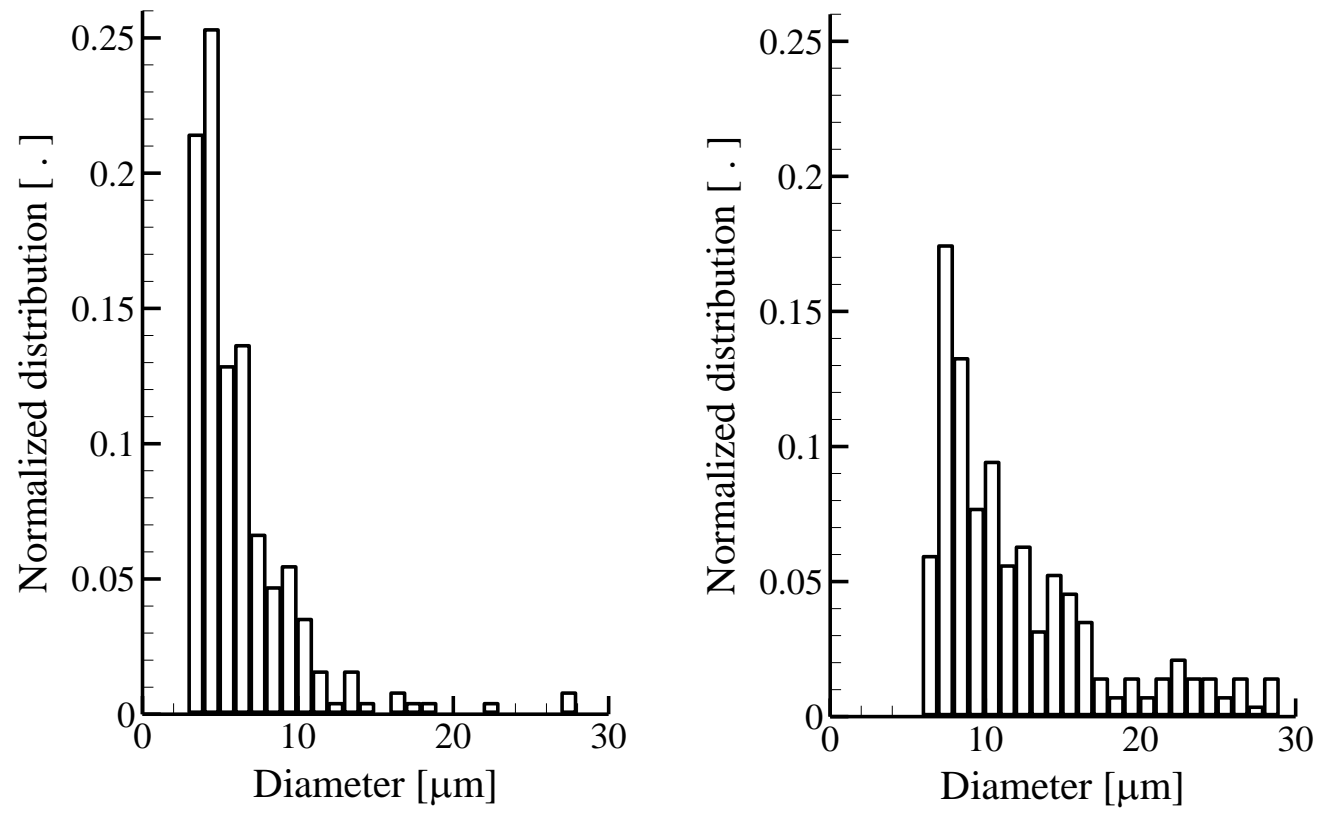

Figure 18. Equivalent diameter distribution at the beginning of injection $(t=6 \mu \mathrm{s})$ from $2 \Delta \mathrm{x}$ to $40 \mu \mathrm{m}$. Left panel: $\Delta \mathrm{x}=1.66 \mu \mathrm{m}$; Right panel: $\Delta \mathrm{x}=3.32 \mu \mathrm{m}$. 


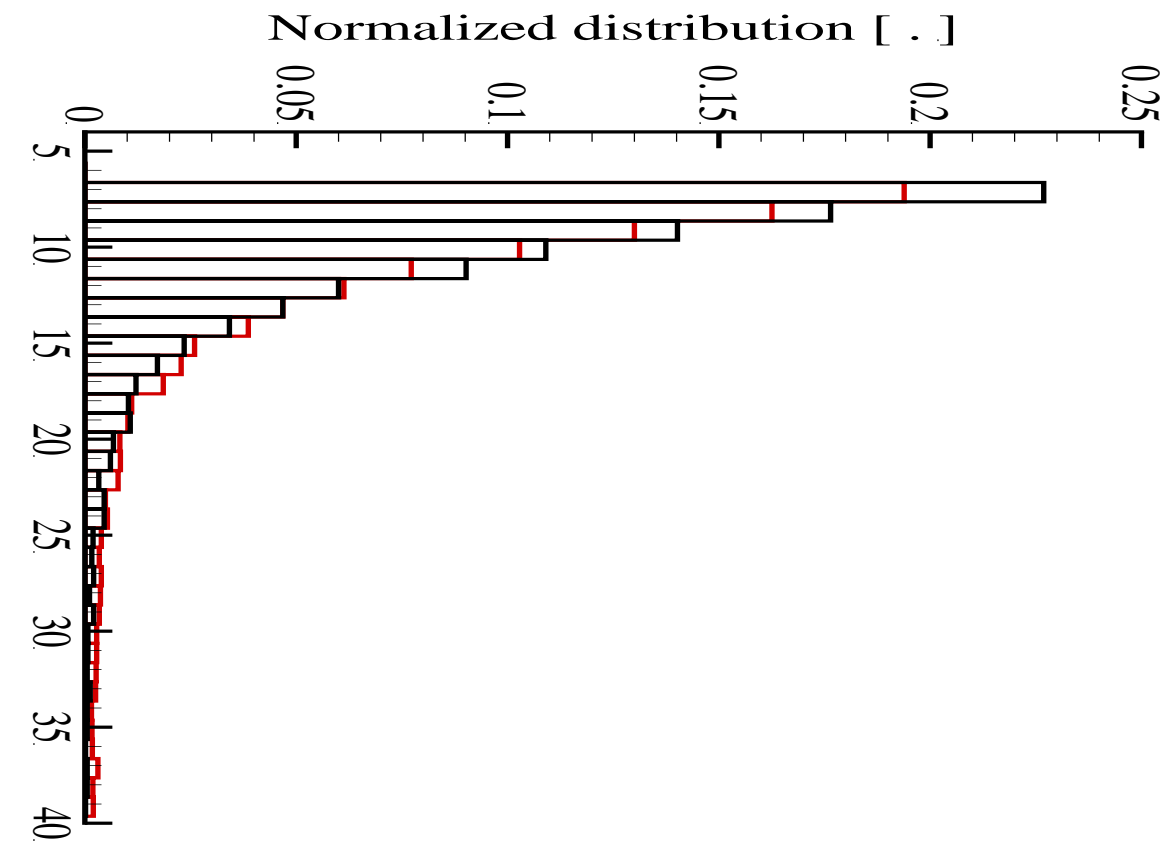

Figure 19. Size distribution for adiabatic wall simulation (red line), at $t=21.2 \mu \mathrm{s}$ from ASOI and isothermal wall (black line) simulation at $t=19.6 \mu$ s from ASOI. 


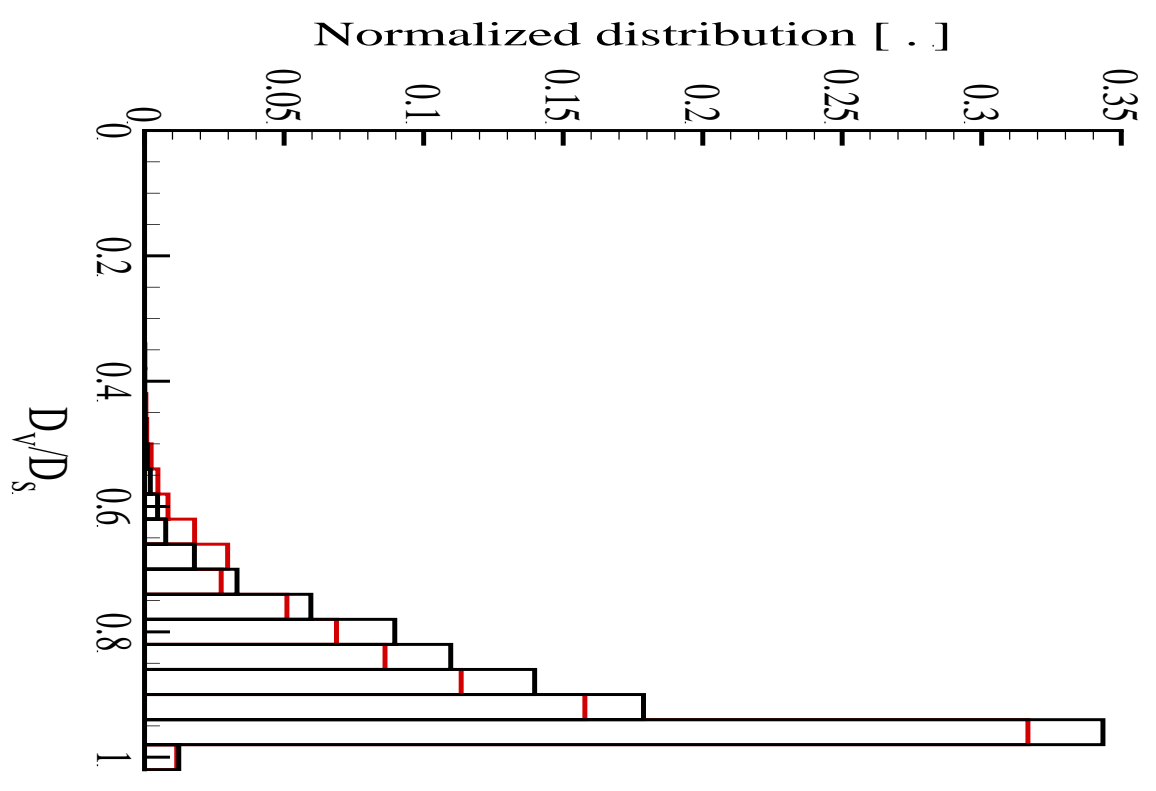

Figure 20. Normalized distribution of the ratio between volume-equivalent and surface-equivalent diameters for spray at adiabatic wall conditions (red line) and isothermal wall conditions (black). 


\section{REFERENCES}

1. Arienti, M., Pan, W., Li, X. and Karniadakis, G. "Many-body Dissipative Particle Dynamics Simulation of Liquid/Vapor and Liquid/Solid Interactions." J. Chem. Phys. 134: 204114 (2011).

2. Arienti, M. and Sussman, M., "Nozzle Geometry Effects on Primary Atomization." ICLASS 2012, $12^{\text {th }}$ Triennial International Conference on Liquid Atomization and Spray Systems, Heidelberg, Germany.

3. Arienti, M., Li, X., Soteriou, M. C., Eckett, C. A., Sussman, M., and Jensen, R. J., "Coupled Level-Set/Volume-of-Fluid Method for the Simulation of Liquid Atomization in Propulsion Device Injectors." J. of Propulsion and Power 29(1): 147-157 (2013).

4. Arienti, M. and Sussman, M., "An Embedded Level Set Method for Sharp-Interface Multiphase Simulations of Diesel Injectors." Int. J. of Multiphase Flow 59: 1-14 (2014).

5. Arienti, M. and Sussman, M., "A High-Fidelity Study of High-Pressure Diesel Injection." SAE Technical Paper 2015-01-1853 (2015).

6. Arienti, M., Doisneau F., and Oefelein, J., "Computation of the break-up of a molten metal drop under sudden acceleration" ILASS Americas $28^{\text {th }}$ Annual Conference on Liquid Atomization and Spray Systems, Dearborn, MI, May 2016. 
7. Battistoni, M., Qingluan, X., and Sibendu, S. "Large-Eddy Simulation (LES) of Spray Transients: Start and End of Injection Phenomena." Oil Gas Sci. Technol. - Rev. IFP Energies nouvelles, 711 (2015).

8. Bode, M., Diewald, F., Broll, D., Heyse, J., le Chenadec, V., and Pitsch, H., "Influence of the Injector Geometry on Primary Breakup in Diesel Injector Systems," SAE Technical Paper 2014-01-1427, 2014.

9. Caudwell, D.R., Trusler, J.P.M., Vesovic, V. and Wakeham, W.A., "The Viscosity and Density of n-Dodecane and n-Octadecane at Pressures up to $200 \mathrm{MPa}$ and Temperatures up to 473 K." International Journal of Thermophysics, Vol. 25, No. 5, 2004.

10. CCSE 2012 at https://ccse.lbl.gov/BoxLib/index.html

11. CMT www.cmt.upv.es/ECN09.aspx accessed on Feb. 27, 2015.

12. Crua, C., de Sercey, G., and Heikal, M. R. "Dropsizing of Near-Nozzle Diesel and RME Sprays by Microscopic Imaging." ICLASS 2012, 12th Triennial International Conference on Liquid Atomization and Spray Systems, Heidelberg, Germany.

13. ECN2 Proceedings, Sept. 2012. http://www.sandia.gov/ecn/workshop/ECN2.php

14. ECN 2013. http://www.sandia.gov/ecn/cvdata/targetCondition/injectorNozGeom.php

15. ECN4 Proceedings, Sept. 2015. http://www.sandia.gov/ecn/workshop/ECN4/ECN4.php 
16. Desjardins O, Moureau V, Knudsen E, Herrmann M, Pitsch H. "Conservative level set/ghost fluid method for simulating primary atomization." ILASS Americas $20^{\text {th }}$ Annual Conference on Liquid Atomization and Spray Systems, Chicago, IL, May 2007.

17. Herrmann, M. (2011) "On Simulating Primary Atomization Using the Refined Level Set Grid Method." Atomization and Sprays, 21(4), 283-301.

18. Herrmann, M., "A parallel Eulerian interface tracking/Lagrangian point particle multi-scale coupling procedure." J. of Computational Physics, 229(3) 745-759, 2010.

19. Jemison, M., Loch, E., Sussman, M., Shashkov, M., Arienti, M., Ohta, M. and Wang, Y. "A Coupled Level Set-Moment of Fluid Method for Incompressible Two-Phase Flows." J. of Scientific Computing, 54:454-491, 2013.

20. Jemison, M., Sussman, M., and Arienti, M., "Compressible, Multi-Phase Semi-Implicit Method with Moment of Fluid Interface Representation." J. of Computational Physics, 279, 182-217, 2014.

21. Jung, Y., Manin, J., Skeen, S., Pickett, L.M., 2015. "Measurement of Liquid and Vapor Penetration of Diesel Sprays with a Variation in Spreading Angle." SAE Technical Paper 2015-01-0946.

22. Kadiouglu, S. Y. and Sussman, M., 2008. "Adaptive Solution Techniques for Simulating Underwater Explosions and Implosions." J. of Comp. Phys. 227:2083-2104. 
23. Kastengren, A. L., Tilocco, F.Z., Powell, C.F. Manin, J., Pickett, L. M., Payri, R., and Bazyn, T. (2012) "Engine Combustion Network (ECN): Measurements of Nozzle Diameter and Hydraulic Behavior of Diesel Sprays." Atomization and Sprays, 22(12) 1011-1052.

24. Kastengren A.L., Tilocco F.Z., Duke D., Powell C.F., Zhang X., Moon S. (2014) "Time-resolved X-ray radiography of sprays from Engine Combustion Network spray A Diesel injectors." Atomization and Sprays 24(3) 251-272.

25. Khasanshin, T. S., Shchamialiou, A. P., and Poddubskij, O. G., "Thermodynamic Properties of Heavy n-Alkanes in the Liquid State: n-Dodecane." International Journal of Thermophysics, Vol. 24, No. 5, 2003.

26. Kook, S. and Pickett, L.M. (2010) "Effect of Ambient Temperature and Density on Diesel-Spray-Generated Shock Waves." Atomization and Sprays, 20(2), 163-175.

27. Kwatra, N., Su, J., Grétarsson, J.T., and Fedkiw, R. (2009) "A method for avoiding the acoustic time step restriction in compressible flow." J. Comp. Phys. 228: 4146-4161.

28. Lemmon, E.W., McLinden, M.O., and Friend D.G. "Thermophysical Properties of Fluid Systems" in NIST Chemistry WebBook, NIST Standard Reference Database Number 69, Eds. P.J. Linstrom and W.G. Mallard, National Institute of Standards and Technology, Gaithersburg MD, 20899, http://webbook.nist.gov (retrieved April 7, 2015). 
29. Meijer, M., Somers, B., Johnson, J., Naber, J., Lee, S-Y, Malbec, L.M., Bruneaux, G., Pickett, L.M., Bardi, M., Payri, R., \& Bazyn, T. (2012) "Engine Combustion Network (ECN): Characterization and comparison of boundary conditions for different combustion vessels." Atomization and Sprays, 22 (9): 777-806.

30. Ménard, T., Tanguy, S. and Berlemont, A., 2007. "Coupling level set/VOF/ghost fluid methods: Validation and application to 3D simulation of the primary break-up of a liquid jet.“ Int. J. of Multiphase Flow 33 510-524.

31. Padilla-Victoria, H., Iglesias-Silva, G. A., Ramos-Estrada, M., Hall K. R., 2013. "A correlation to predict speed of sound in liquids: 1 . n-Alkanes $\left(\geq C_{5}\right)$ and their mixtures at high pressures. " Fluid Phase Equilibria 338 119-127.

32. Payri, R., Salvador, F.J., Gimeno, J., Bracho, G. (2008) "A new methodology for correcting the signal cumulative phenomenon on injection rate measurements." Experimental Techniques 32 (1) 46-49.

33. Pickett, L., Manin, J., Payri, R., Bardi, M., and Gimeno, J., "Transient Rate of Injection Effects on Spray Development." SAE Technical Paper 2013-24-0001, 2013.

34. Pickett, L., Manin, J., Kastengren, A., and Powell, C., "Comparison of Near-Field Structure and Growth of a Diesel Spray Using Light-Based Optical Microscopy and X-Ray Radiography," SAE Int. J. Engines 7(2): 1044-1053, 2014. 
35. Pilliod, J. and Puckett, E. "Second-order accurate volume-of-fluid algorithms for tracking material interfaces." J. Computational Phys. 199(2), 465-502 (2004).

36. Powell, C. F., Duke, D., Kastengren, A. L., and llavsky, J. "Measurement of Diesel Spray Droplet Size with Ultra-Small Angle X-Ray Scattering." ILASS Americas, 25th Annual Conference on Liquid Atomization and Spray Systems, Pittsburgh, PA, May 2013.

37. Queimada, A.J., Marrucho, I.M., Coutinho, J.A.P. "Surface tension of pure heavy n-alkanes: a corresponding states approach," Fluid Phase Equilibria, 4639 1-10 (2001).

38. Salemi, R., McDavid, R., Koukouvinis, P., Gavaises, M., and Marengo, M. (2015). "Steady state and transient, non-isothermal modeling of cavitation in diesel fuel injectors." Proceedings of ILASS Americas $27^{\text {th }}$ Annual Conference on Liquid Atomization and Spray Systems, Raleigh, NC, May.

39. Shinjo, J. and Umemura, A. (2011). "Detailed simulation of primary atomization mechanisms in Diesel jet sprays (isolated identification of liquid jet tip effects)." Proceedings of the Combustion Institute 33 2089-2097.

40. Sussman, M., and Ohta, M. "A Stable and Efficient Method for Treating Surface Tension in Incompressible Two-phase Flow". SIAM J. Sci. Comput., 31(4):2447-2471, 2009.

41. Zisman, A.W., "Relation of the Equilibrium Contact Angle to Liquid and Solid Constitution", Contact Angle, Wettability, and Adhesion. January 1, $1964,1-51$ 
42. Xue, Q., Som, S., Battistoni, M., Longman, D. E. Zhao, H., Senecal, P. K. and Pomraning, E.

"Three-dimensional Simulations of the Transient Internal Flow in a Diesel Injector: Effects of Needle Movement". In ILASS Americas, 25th Annual Conference on Liquid Atomization and Spray Systems, Pittsburgh, PA, May 2013. 